TRANSACTIONS OF THE

AMERICAN MATHEMATICAL SOCIETY

Volume 357, Number 11, Pages 4269-4299

S 0002-9947(05)04034-1

Article electronically published on June 21, 2005

\title{
FIRST COUNTABLE, COUNTABLY COMPACT SPACES AND THE CONTINUUM HYPOTHESIS
}

\author{
TODD EISWORTH AND PETER NYIKOS
}

\begin{abstract}
We build a model of $\mathrm{ZFC}+\mathrm{CH}$ in which every first countable, countably compact space is either compact or contains a homeomorphic copy of $\omega_{1}$ with the order topology. The majority of the paper consists of developing forcing technology that allows us to conclude that our iteration adds no reals. Our results generalize Saharon Shelah's iteration theorems appearing in Chapters V and VIII of Proper and improper forcing (1998), as well as Eisworth and Roitman's (1999) iteration theorem. We close the paper with a ZFC example (constructed using Shelah's club-guessing sequences) that shows similar results do not hold for closed pre-images of $\omega_{2}$.
\end{abstract}

\section{INTRODUCTION}

In general topology, the study of the relationship between compactness ("every open cover has a finite subcover") and countable compactness ("every infinite set has a point of accumulation") has a distinguished history reaching back to the earliest days of the subject. It has long been known that these two concepts are not the same, even in the class of first countable spaces (i.e., spaces in which every point has a countable neighborhood base). The simplest example of this is the topological space obtained by taking $\omega_{1}$, the first uncountable ordinal, and giving it the natural order topology. The space is easily seen to be first countable, and every infinite subset has a limit point essentially because strictly increasing sequences of countable length converge to their supremum in this topology. The space $\omega_{1}$ is definitely not compact, as the sets of the form $[0, \alpha+1)$ form an open cover with no finite subcover.

This natural example brings to mind the question of to what extent must a noncompact first countable, countably compact space resemble $\omega_{1}$. A more precise formulation of this is "is it true that every first countable, countably compact space is either compact, or contains a closed subset homeomorphic to $\omega_{1}$ ?". This question first appeared in print at least 15 years ago - it was explicitly asked by the second author in the article [14] because it has some bearing on the theory of non-metrizable manifolds.

This answer to this question was found to be independent of ZFC, the usual axioms of set theory. The second author showed that a very weak form of the axiom $\diamond$ (so weak that it is compatible with Martin's Axiom) yields a counterexample (see

Received by the editors May 23, 2002.

2000 Mathematics Subject Classification. Primary 03E75.

Key words and phrases. Proper forcing, iterations, Continuum Hypothesis, pre-images of $\omega_{1}$.

The first author was partially supported by a Summer Fellowship granted by the Graduate College of the University of Northern Iowa. 
Fremlin's article 11] for a proof), while the consistency of a positive answer was a byproduct of the torrent of consistency results obtained in the 1980's by Balogh, Dow, Fremlin, and Nyikos ([1], [2], [11]) concerning the Proper Forcing Axiom (PFA) and its consequences for topology.

The goal of this paper is to show that the Continuum Hypothesis does not decide the answer to the question - we produce a model of ZFC in which the Continuum Hypothesis holds and in which first countable, countably compact spaces are either compact or contain a closed subset homeomorphic to $\omega_{1}$. The results and method contained in this paper have had some impact on current research in set-theoretic topology. For example, Gruenhage [13] has shown that in the model we produce, all countably compact spaces with small diagonal are compact. The notion of forcing we define in the paper has been further generalized by the first author [7] to show that PFA implies that countably tight perfect pre-images of $\omega_{1}$ contain copies of $\omega_{1}$, while the iteration technology developed here is crucial for the first author's work on $\mathrm{CH}$ and the Moore-Mrówka problem (see [8]).

Our argument is organized along the following lines. In section 2, we show that first countable closed pre-images of $\omega_{1}$ of size $\omega_{1}$ behave a lot like $\omega_{1}$ itself - there are natural analogs of the closed unbounded filter and a lot of the properties of the club filter carry over into this more general context. In section 3, we define a notion of forcing that shoots a copy of $\omega_{1}$ through a given first countable closed pre-image of $\omega_{1}$. In sections 4 and 5 we define weakly $<\omega_{1}$-proper notions of forcing, verify that the notion of forcing defined in section 3 is weakly $<\omega_{1}$-proper, and prove that this property is preserved by countable support iterations. In section 6 we use weakly $<\omega_{1}$-properness to prove an iteration theorem useful in constructing models of $\mathrm{ZFC}+\mathrm{CH}$. In section [] we construct a model of $\mathrm{ZFC}+\mathrm{CH}+$ "first countable, countably compact spaces are either compact or contain a copy of $\omega_{1}$ ". Finally, in the last section we show that $\omega_{1}$ is different from $\omega_{2}$ in that there is a closed two-to-one pre-image of $\omega_{2}$ that does not contain a copy of $\omega_{2}$. The proof uses one of the basic results of Shelah's theory of guessing clubs.

We make some demands regarding the reader's background. In particular, we assume that the reader is comfortable with proper forcing and elementary submodels, as well as their use in topology. Those who are familiar with Dow's papers [3], [4], and 5] should have no trouble with the topological portion of the paper, while Goldstern's [12] provides an excellent background for the theory of iterated forcing. A detailed treatment of totally proper forcing can be found in [9]. A reader needing more background on the topological concepts involved should check out Engelking's encyclopedic reference [10], or Vaughan's article [20] on countable compactness and related ideas.

\section{BASICS}

In this section, we study the structure of first countable topological spaces that can be mapped by a closed continuous function onto the space $\omega_{1}$. In particular, we show that such spaces have much in common with $\omega_{1}$ - one can define analogs of "closed unbounded" and "stationary".

Let $X$ be a first countable topological space for which there is a closed (continuous) mapping $\pi: X \rightarrow \omega_{1}$, and let $\mathcal{U}$ be a maximal filter of closed sets that contains $\pi^{-1}(C)$ for every closed unbounded $C \subseteq \omega_{1}$. 
We assume that $X$ has size $\aleph_{1}$. If $\mathrm{CH}$ holds, then every first countably closed pre-image of $\omega_{1}$ contains a closed subspace with the same properties that is also of size $\aleph_{1}$, so this assumption causes no loss of generality for our purposes.

We will show that the filter $\mathcal{U}$ on $X$ behaves much as the filter of closed unbounded subsets of $\omega_{1}$ does. It is not hard to see that $\mathcal{U}$ is closed under countable intersections (this is done in Proposition 2.2 below); we also show that it is possible to define an analog of diagonal intersections, and show that $\mathcal{U}$ is closed under such intersections. The combinatorics exposed in this section are the key to proving that the notion of forcing defined in the next section is proper and does not add reals.

Proposition 2.1. If $\left\{x_{n}: n \in \omega\right\} \subseteq X$ is such that $\left\{\pi\left(x_{n}\right): n \in \omega\right\}$ is increasing, then $\left\{x_{n}: n \in \omega\right\}$ has a limit point $x$ with $\pi(x)=\sup \left\{\pi\left(x_{n}\right): n \in \omega\right\}$

Proof. Immediate, because the mapping $\pi$ is closed.

Proposition 2.2. $\mathcal{U}$ is countably closed.

Proof. Let $\left\{A_{n}: n \in \omega\right\} \subseteq \mathcal{U}$ be given. We may assume that $A_{n} \supseteq A_{n+1}$. Choose $x_{n} \in A_{n}$ such that the sequence $\left\{\pi\left(x_{n}\right): n \in \omega\right\}$ is strictly increasing. The sequence of $x_{n}$ 's has a limit point $x$ (by the previous proposition), and $x$ witnesses that $\bigcap\left\{A_{n}: n \in \omega\right\}$ is non-empty. A similar proof shows that this intersection is not disjoint to any set in $\mathcal{U}$, so by maximality of $\mathcal{U}$ it must be a member of $\mathcal{U}$.

Our next project is to define a version of diagonal intersection that is appropriate to this context, and prove that our filter $\mathcal{U}$ is closed under such intersections.

Definition 2.3. Let $N$ be a countable elementary submodel of $H(\lambda)$ that contains $X$ and $\mathcal{U}$. We define the trace of $N$, denoted $\operatorname{Tr}(N)$, by

$$
\operatorname{Tr}(N)=\bigcap_{A \in N \cap \mathcal{U}} \operatorname{cl}(A \cap N)
$$

Proposition 2.4. Let $N$ be a countable elementary submodel of $H(\lambda)$ containing $X$ and $\mathcal{U}$.

(1) $\operatorname{Tr}(N)$ is a non-empty closed set, and $\pi \uparrow \operatorname{Tr}(N)$ is constant with value $N \cap \omega_{1}$.

(2) If $\left\{N_{\alpha}: \alpha<\omega_{1}\right\}$ is a continuously increasing $\in$-chain of countable elementary submodels of $H(\lambda)$, then

$$
\bigcup_{\alpha<\omega_{1}} \operatorname{Tr}\left(N_{\alpha}\right) \in \mathcal{U}
$$

Proof. For the first part, let $\left\{A_{n}: n \in \omega\right\}$ enumerate $N \cap \mathcal{U}$. For each $n \in \omega$, select a point $x_{n} \in N \cap A_{0} \cap \cdots \cap A_{n}$ in such a way that $\pi\left(x_{n}\right)<\pi\left(x_{n+1}\right)$. The sequence $\left\{x_{n}: n \in \omega\right\}$ has a limit point $x$ that is in $\operatorname{Tr}(N)$ by definition. $\operatorname{Tr}(N)$ is closed by its definition, and the statement about $\pi \uparrow \operatorname{Tr}(N)$ also follows easily.

To establish the second part, it suffices to show that the set in (2.2) is closed, and that it is not disjoint to any set in $\mathcal{U}$.

Let $Z$ be the set defined in (2.2). Since $X$ is first countable, to show that $Z$ is closed it suffices to prove that if $\left\{x_{n}: n \in \omega\right\}$ is a sequence of points from $Z$ that converge to a point $x$, then $x \in Z$.

Suppose we are given such a sequence $\left\{x_{n}: n \in \omega\right\}$. By passing to a subsequence, we may assume that the sequence $\left\{\pi\left(x_{n}\right): n \in \omega\right\}$ is either constant, or strictly 
increasing. In the former case, we are done by the first part of the proposition, so assume the latter case occurs.

Let $\beta_{n}$ be the unique ordinal with $x_{n} \in \operatorname{Tr}\left(N_{\beta_{n}}\right)$, and let $\beta=\sup \left\{\beta_{n}: n \in \omega\right\}$. It suffices to show that $x \in \operatorname{Tr}\left(N_{\beta}\right)$, i.e., if $A \in N_{\beta} \cap \mathcal{U}$ is arbitrary, then $x \in \operatorname{cl}\left(N_{\beta} \cap A\right)$.

Note that $\beta$ is a limit ordinal and so $N_{\beta}=\bigcup\left\{N_{\alpha}: \alpha<\beta\right\}$. Since the sequence of $N_{\alpha}$ 's is increasing, we have that $A \in N_{\beta_{n}} \cap \mathcal{U}$ for all but finitely many $n$. This means that for all large enough $n$

$$
x_{n} \in \operatorname{cl}\left(N_{\beta_{n}} \cap A\right) \subseteq \operatorname{cl}\left(N_{\beta} \cap A\right),
$$

and so $x \in \operatorname{cl}\left(N_{\beta} \cap A\right)$ as required.

Now that we have shown that $Z$ is closed, to show that $Z \in \mathcal{U}$ it suffices to take an arbitrary $B \in \mathcal{U}$ and prove that $Z \cap B \neq \emptyset$. Given such a $B$, choose countable $M \prec H(\lambda)$ containing $X, Z, \mathcal{U}, B$, and $\left\{N_{\alpha}: \alpha<\omega_{1}\right\}$.

If $\delta=M \cap \omega_{1}$, then $\delta=N_{\delta} \cap \omega_{1}$ as well. Note also that $N_{\delta} \subseteq M$ by the properties of the sequence $\left\{N_{\alpha}: \alpha<\omega_{1}\right\}$, and clearly since $|X|=\aleph_{1}$, for $A \in N_{\delta} \cap \mathcal{U}$ we have $N_{\delta} \cap A=M \cap A$. Thus we have $\operatorname{Tr}(M) \subseteq \operatorname{Tr}\left(N_{\delta}\right)$. Since $\operatorname{Tr}(M)$ is a non-empty subset of $B$, any $x \in \operatorname{Tr}(M) \subseteq \operatorname{Tr}\left(N_{\delta}\right)$ witnesses that $Z \cap B$ is non-empty.

Definition 2.5. Call a subset $Y$ of $X$ large if it meets every set in $\mathcal{U}$. Otherwise, $Y$ is said to be small.

Note that in light of the fact that $\mathcal{U}$ is countably complete, we have that a countable union of small sets is small.

Definition 2.6. A promise is a function $f$ such that

- $\operatorname{dom} f$ is a large subset of $X$,

- $f(x)$ is an open neighborhood of $x$ for $x \operatorname{dom} f$.

Definition 2.7. A point $y \in X$ is banned by a promise $f$ if $\{x \in \operatorname{dom} f: y \in f(x)\}$ is small. Let Ban $f$ be the set of all points $y$ that are banned by $f$.

Proposition 2.8. If $y$ is not banned by a promise $f$, then there is an open neighborhood $U$ of $y$ for which

$$
\{x \in \operatorname{dom} f: U \subseteq f(x)\} \text { is large. }
$$

Thus Ban $f$ is closed.

Proof. Let $\left\{U_{n}: n \in \omega\right\}$ be a neighborhood base for $y$. If $y \in f(x)$, then there is an $n$ for which $U_{n} \subseteq f(x)$. Since a countable union of small sets is small, there must be a single $n$ for which $\left\{x \in \operatorname{dom} f: U_{n} \subseteq f(x)\right\}$ is large, as desired.

Proposition 2.9. Ban $f$ is small.

Proof. If not, then we know $\operatorname{Ban} f \in \mathcal{U}$ as it is a closed set. For each $y \in \operatorname{Ban} f$, choose a set $A_{y} \in \mathcal{U}$ disjoint to $\{x \in \operatorname{dom} f: y \in f(x)\}$. Let $\left\{N_{\alpha}: \alpha<\omega_{1}\right\}$ be a tower of models as in the second part of Proposition 2.4 chosen so that both $f$ and the function sending $y \in \operatorname{Ban} f$ to $A_{y}$ are in $N_{0}$.

Since $\operatorname{dom} f$ is large, by Proposition 2.4 there is an $\alpha$ and $x \in \operatorname{dom} f$ such that $x \in \operatorname{Tr}\left(N_{\alpha}\right)$. This means that $x \in A_{y}$ for all $y \in N_{\alpha} \cap \operatorname{Ban} f$ and $x \in \operatorname{cl}\left(\operatorname{Ban} f \cap N_{\alpha}\right)$. This latter point means that $f(x)$ must contain a point $y \in \operatorname{Ban} f \cap N_{\alpha}$ and this contradicts the fact that $x \in A_{y}$ for this $y$. 


\section{A NOTION OF FORCING}

In this section, we will define a notion of forcing that will shoot a copy of $\omega_{1}$ into a given first countable closed pre-image of $\omega_{1}$. This notion of forcing is proper, and it adds no reals. Before we begin, we need some definitions from $[9]$ and $[6]$.

Definition 3.1. Let $P$ be a notion of forcing, and let $N$ be a countable elementary submodel of $H(\lambda)$ for some large enough regular $\lambda$. We say a condition $q \in P$ is totally $(N, P)$-generic if whenever $D$ is a dense open subset of $P$ that is in $N$, we can find a condition $p \in N \cap D$ with $q \leq p$. We say that $P$ is totally proper if, given $N$ as above, every $p \in N \cap P$ has a totally $(N, P)$-generic extension $q$.

Definition 3.2. Define a notion of forcing $P=P_{X}$ by putting $p$ into $P$ if and only if $p=\left([p], \Phi_{p}\right)$, where

(1) $[p]$ is a countable closed subset of $X$,

(2) $\Phi_{p}$ is a countable collection of promises,

(3) $\pi \uparrow[p]$ is one-to-one.

A condition $q$ extends $p$ if

(4) $[q]$ "end extends" $[p]$, i.e., if $x \in[q] \backslash[p]$, then $\pi(x)>\max \operatorname{ran}(\pi \uparrow[q])$,

(5) $\Phi_{q} \supseteq \Phi_{p}$,

(6) for each promise $f \in \Phi_{p}$ the set

$$
Y(f, q, p)=\{x \in \operatorname{dom} f:[q] \backslash[p] \subseteq f(x)\}
$$

is large, and $f \uparrow Y(f, q, p) \in \Phi_{q}$.

Definition 3.3. Given $p \in P$ and $D \subseteq P$ dense open, we say a point $x \in X$ is bad for $p$ and $D$ if $x$ has an open neighborhood $U_{x}$ for which there is no $q \leq p$ with $q \in D$ and $[q] \backslash[p] \subseteq U_{x}$. We let $\operatorname{Bad}(p, D)$ be the set of all points $x$ that are bad for $p$ and $D$.

Proposition 3.4. If $p \in P$ and $D \subseteq P$ is dense open, then $\operatorname{Bad}(p, D)$ is small.

Proof. Suppose not. Then the function $f$ with domain $\operatorname{Bad}(p, D)$ that sends $x$ to the neighborhood $U_{x}$ from the previous definition is a promise. Let $q=\left([q], \Phi_{p} \cup\{f\}\right)$; clearly $q$ extends $p$ in $P$. Since $D$ is dense, there is an extension $r \leq q$ with $r \in D$. By definition, this means

$$
Y(f, r, q)=\{x \in \operatorname{dom} f:[r] \backslash[q] \subseteq f(x)\}
$$

is large. In particular, it is non-empty and this is a contradiction, for if $x$ is in $Y(f, r, q)$, then $[r] \backslash[q]=[r] \backslash[p]$ is contained in $f(x)=U_{x}$.

Theorem 1. Let $N$ be a countable elementary submodel of $H(\lambda)$ containing $X$ and $P$, and let $p \in P$ be arbitrary. Further assume $\bar{x} \in \operatorname{Tr}(N)$ and $U$ is a neighborhood of $\bar{x}$. Then there is a totally $(N, P)$-generic $q \leq p$ with $[q] \backslash[p] \subseteq U$. In particular, $P$ is totally proper.

Proof. Let $\left\{D_{n}: n \in \omega\right\}$ list the dense open subsets of $P$ that are in $N$. Let $\left\{U_{n}: n \in \omega\right\}$ be a decreasing neighborhood base for $\bar{x}$ with $U_{0}=U$. We define by induction on $n$ a sequence $\left\{p_{n}: n \in \omega\right\}$ and function $h \in^{\omega} \omega$ such that
(a) $p_{0}=p, h(0)=0$,
(b) $p_{n+1} \leq p_{n}$
(c) $p_{n+1} \in N \cap D_{n}$,
(d) $h(n)<h(n+1)$, 
(e) $\left[p_{n+1}\right] \backslash\left[p_{n}\right] \subseteq U_{h(n+1)}$,

(f) if $f$ is a promise in $\Phi_{i}$ for some $i$, then there is a stage $n \geq i$ for which

$$
K(f, n, i)=\left\{x \in Y\left(f, p_{n}, p_{i}\right): U_{h(n+1)} \subseteq f(x)\right\}
$$

is large.

Claim 3.5. If we can carry out the above construction, then $\left\{p_{n}: n<\omega\right\}$ has a lower bound $q$ with $[q] \backslash[p] \subseteq U$.

Proof of Claim. Let $[q]=\bigcup_{n \in \omega}\left[p_{n}\right] \cup\{\bar{x}\}$.

Subclaim 1. $[q]$ is closed.

Proof of Subclaim 1. Let $\left\{z_{k}: k \in \omega\right\} \subseteq[q]$ converge to a point $z$. If there is an $n$ so that $z_{k} \in\left[p_{n}\right]$ for infinitely many $k$, then $z \in\left[p_{n}\right] \subseteq[q]$ as well because $\left[p_{n}\right]$ is closed. If no such $n$ exists, then conditions (d) and (e) of our construction guarantee that the sequence of $z_{k}$ 's converges to $\bar{x}$, so $z=\bar{x} \in[q]$ as required.

Clearly we have that $\pi\lceil[q]$ is one-to-one, and $[q] \backslash[p] \subseteq U$ as well.

Subclaim 2. If $f \in \Phi_{p_{i}}$ for some $i$, then

$$
Y\left(f, q, p_{i}\right):=\left\{x \in \operatorname{dom} f:[q] \backslash\left[p_{i}\right] \subseteq f(x)\right\}
$$

is large.

Proof of Subclaim 2. Let $n \geq i$ be as in condition (f) for the promise $f$. We will show that $K(f, n, i) \subseteq Y\left(f, q, p_{i}\right)$. To do this, fix $x \in K(f, n, i)$; we must verify that $[q] \backslash\left[p_{i}\right] \subseteq f(x)$. This is trivial, as

$$
\begin{aligned}
{[q] \backslash\left[p_{i}\right] } & =[q] \backslash\left[p_{n}\right] \cup\left[p_{n}\right] \backslash\left[p_{i}\right] \\
& \subseteq U_{h(n+1)} \cup\left[p_{n}\right] \backslash\left[p_{i}\right] \quad \text { by }(\mathrm{e}) \\
& \subseteq U_{h(n+1)} \cup f(x) \quad \text { as } x \in Y\left(f, p_{n}, p_{i}\right) \\
& \subseteq f(x) \cup f(x) \quad \text { by }(\mathrm{f}), \text { as } x \in K(f, n, i) \\
& =f(x) .
\end{aligned}
$$

Now if we define

$$
\Phi_{q}=\bigcup_{n \in \omega} \Phi_{p_{n}} \cup \bigcup_{n \in \omega}\left\{f \uparrow Y\left(f, q, p_{n}\right): n \in \omega\right\},
$$

then $q:=\left([q], \Phi_{q}\right)$ is as advertised.

Now how do we carry out the construction? We assume that some bookkeeping procedure has been fixed at the start so that at each stage we will be handed a promise $f$ for which we must ensure condition (f), and such that every promise appearing along the way will be so considered.

The case where $n=0$ is trivial, so assume we are given $p_{n}$ and $h\lceil n+1$ and that our bookkeeping hands us $f \in \Phi_{p_{i}}$ for some $i \leq n$.

Let $f^{\prime}=f\left\lceil Y\left(f, p_{n}, p_{i}\right)\right.$. Clearly $f^{\prime}$ is a promise and $f^{\prime} \in N$. Since Ban $f^{\prime} \in N$ is small, we know that $\bar{x} \notin \operatorname{Ban} f^{\prime}-$ it is a consequence of the fact that $\bar{x} \in \operatorname{Tr}(N)$. Proposition 2.8 tells us we can choose $h(n+1)>h(n)$ large enough so that

$$
K(f, n, i)=\left\{x \in Y\left(f, p_{n}, p_{i}\right): U_{h(n+1)} \subseteq f(x)\right\}
$$

is large. Thus we have taken care of (f) for the promise $f$.

Now the set $\operatorname{Bad}\left(p_{n}, D_{n}\right)$ is a small set in $N$ (by Proposition [3.4), so we can find $A \in N \cap \mathcal{U}$ disjoint to it. Since $x \in \operatorname{Tr}(N)$, we know that $x \in \operatorname{cl}(N \cap A)$, and 
so there is a point $z \in N \cap U_{h(n+1)}$ such that $z \notin \operatorname{Bad}\left(p_{n}, D_{n}\right)$. Since $X$ is first countable, $z$ has a neighborhood $V$ in $N$ with $V \subseteq U_{h(n+1)}$. Thus we can apply the definition of $z \notin \operatorname{Bad}\left(p_{n}, D_{n}\right)$ inside of $N$ to get $p_{n+1} \leq p_{n}$ in $N \cap D_{n}$ with

$$
\left[p_{n+1}\right] \backslash\left[p_{n}\right] \subseteq V \subseteq U_{h(n+1)} .
$$

This choice of $p_{n+1}$ satisfies conditions (b)-(e), so we have successfully carried out the construction at stage $n+1$. In light of Claim 3.5. we have now given a proof of the theorem.

\section{WEAK $<\omega_{1}$-PROPERNESS}

In this section, we begin dealing with some rather technical conditions that will guarantee that we are able to use iterated forcing to produce a model of ZFC in which the Continuum Hypothesis holds.

The following definition pins down some notation that we will be utilizing throughout the rest of the paper.

Definition 4.1. Let $\lambda$ be some large regular cardinal, and let $x \in H(\lambda)$ be arbitrary. A sequence $\mathfrak{N}=\left\langle N_{\beta}: \beta \leq \alpha\right\rangle$ is said to be an $x$-suitable $\alpha$-tower of models if

- $N_{\beta}$ is a countable elementary submodel of $H(\lambda)$ for $\beta \leq \alpha$,

- $x \in N_{0}$,

- $\left\langle N_{\gamma}: \gamma \leq \beta\right\rangle \in N_{\beta+1}$ for $\beta<\alpha$,

- $\mathfrak{N}$ is continuous at limit ordinals.

We will usually suppress reference to the parameter $x$ in the above definition typically $x$ will be the notion of forcing under discussion.

Definition 4.2. Let $P$ be a notion of forcing, and let $\mathfrak{N}$ be a relevant tower of models. Let $\operatorname{Spec}\left(\mathfrak{N}, \dot{G}_{P}\right)$ be some fixed $P$-name for the set (in the generic extension) of ordinals $i \leq \alpha$ for which $N_{i}\left[\dot{G}_{P}\right] \cap$ Ord $=N_{i} \cap$ Ord. If $G \subseteq P$ is generic, then $\left\{i \leq \alpha: N_{i}[G] \cap\right.$ Ord $=N_{i} \cap$ Ord $\}$ is called the spectrum of genericity of $G$ with respect to $\mathfrak{N}$.

Definition 4.3. Given $\alpha<\omega_{1}$, a notion of forcing $P$ is said to be weakly $\alpha$-proper if whenever $\mathfrak{N}$ is a suitable $\alpha$-tower and $p \in N_{0} \cap P$, there is a $q \leq p$ such that $q$ is $\left(N_{\alpha}, P\right)$-generic, and

$$
q \Vdash \operatorname{Spec}(\mathfrak{N}, \dot{G}) \text { has order-type } \alpha+1 .
$$

Such a $q$ is said to be weakly $(\mathfrak{N}, P)$-generic. If $P$ is weakly $\beta$-proper for all $\beta \leq \alpha$, then we say $P$ is weakly $\leq \alpha$-proper. We say $P$ is weakly $<\omega_{1}$-proper if it is weakly $\alpha$-proper for all $\alpha<\omega_{1}$.

Note that (4.1) is significantly weaker than the statement

$$
\left\{\beta \leq \alpha: q \text { is }\left(N_{\beta}, P\right) \text {-generic }\right\} \text { has order-type } \alpha+1
$$

as it is possible for a condition $q$ to satisfy (4.1) without being $\left(N_{\beta}, P\right)$-generic for some specific $\beta<\alpha$. We use (4.1) instead of (4.2) in our formulation of weak $\alpha$-properness so that we can prove iteration theorems - the corresponding formulation of weak $\alpha$-properness based on (4.2) is not even preserved by iterations of length two. 
Definition 4.4. Let $\mathfrak{N}=\left\langle N_{i}: i \leq \alpha\right\rangle$ be a suitable tower. We say that $I \subseteq \alpha+1$ is an $\mathfrak{N}$-suitable index set if $I$ is closed in $\alpha$ and for $\beta \in I$,

$$
I \cap \beta \in N_{\beta+1} .
$$

If the tower $\mathfrak{N}$ is clear from the context, then we will often say only that $I$ is a suitable index set.

Proposition 4.5. Let $P$ be a notion of forcing, and let $\mathfrak{N}=\left\langle N_{i}: i \leq \alpha\right\rangle$ be a suitable tower.

(1) If $I \subseteq \alpha$ is a suitable index set, then $\mathfrak{N} \uparrow I=\left\langle N_{i}: i \in I\right\rangle$ is a suitable tower.

(2) $\Vdash_{P}$ "Spec $\left(\mathfrak{N}, \dot{G}_{P}\right)$ is an $\mathfrak{N}[G]$-suitable index set".

Proof. The first statement follows immediately from the definition. Regarding the notation of the second statement, if $G \subseteq P$ is generic, then

$$
\mathfrak{N}[G]:=\left\langle N_{i}[G]: i \leq \alpha\right\rangle .
$$

Let $G$ be an arbitrary generic subset of $P$. Previously known results (see Theorem 2.11 of Chapter III in [19]) tell us that $\mathfrak{N}[G]$ is a suitable tower in $V[G]$. Let $I$ denote the interpretation of the $P-$ name $\operatorname{Spec}\left(\mathfrak{N}, \dot{G}_{P}\right)$. Since $\mathfrak{N}[G]$ is continuous at limit ordinals, it follows that $I$ is a closed subset of $\alpha+1$. For any $\beta \leq \alpha, I \cap \beta$ is definable from $\mathfrak{N} \uparrow \beta$ and $G$. Since both $G$ and $\mathfrak{N} \uparrow \beta$ are elements of $N_{\beta+1}[G]$, it follows that $I \cap \beta \in N_{\beta+1}[G]$ as required.

The following lemma has a fairly easy proof that is left to the reader - it is simply a version of III.3.3.G in [19] and it is proved in the same fashion.

Lemma 4.6. Let $P * \dot{Q}$ be a forcing iteration of length two, and let $\mathfrak{N}=\left\langle N_{i}: i \leq \alpha\right\rangle$ be a suitable tower. A condition $p * \dot{q} \in P * \dot{Q}$ is weakly $(\mathfrak{N}, P * \dot{Q})$-generic if and only if $p$ is weakly $(\mathfrak{N}, P)$-generic and

$$
p \Vdash \dot{q} \text { is weakly }\left(\mathfrak{N}[G] \uparrow \operatorname{Spec}\left(\mathfrak{N}, \dot{G}_{P}\right), \dot{Q}\right) \text {-generic. }
$$

Notice that (4.5) is stronger than requiring that $p$ forces $\dot{q}$ to be weakly $(\mathfrak{N}[G], \dot{Q})-$ generic - it can be shown that the lemma is false once this change is made. However, the lemma is strong enough to prove the following corollary.

Corollary 4.7. If $P$ is weakly $\alpha$-proper and $\dot{Q}$ is a $P$-name for a weakly $\alpha$-proper notion of forcing, then $P * \dot{Q}$ is weakly $\alpha$-proper.

In the next section, we will prove a full-fledged iteration theorem for weakly $<\omega_{1}$-proper notions of forcing.

We now show that the concept of weak $<\omega_{1}$-properness has some relevance to our topological question by verifying that the notion of forcing from section 3 is weakly $<\omega_{1}$-proper. In the introduction, we mentioned that Fremlin's paper [11] contains a simple construction (assuming the axiom $\diamond$ ) of a first countable perfect pre-image of $\omega_{1}$ that contains no copy of $\omega_{1}$; for this particular space, the notion of forcing we have been looking at fails to be $\omega$-proper.

Definition 4.8. We call an ordinal $\alpha<\omega_{1}$ good if whenever $\mathfrak{N}=\left\langle N_{i}: i \leq \alpha\right\rangle$ is a relevant $\alpha$-tower, there is a point $\bar{x} \in \operatorname{Tr}\left(N_{\alpha}\right)$ such that for every $p \in N_{\alpha} \cap P$ and open neighborhood $U$ of $\bar{x}$, there is a $q \leq p$ such that

- $q$ is totally $\left(N_{\alpha}, P\right)$-generic, 
- $q$ is weakly $(\mathfrak{N}, P)$-generic,

- $[q] \backslash[p] \subseteq U$.

Lemma 4.9. Let $\alpha<\omega_{1}$ be an infinite ordinal that is closed under addition. Then there is a sequence $\left\{\alpha_{n}: n \in \omega\right\}$ of ordinals such that

(1) $\alpha=\sup _{n \in \omega} \alpha_{n}$,

(2) $\alpha_{n}<\alpha_{n+1}$,

(3) $\operatorname{otp}\left(\alpha_{n+1} \backslash \alpha_{n}\right)$ is closed under addition.

Proof. Let $C \subseteq \omega_{1}$ be the set of countable ordinals that are closed under addition (including the ordinal 1). Note that $C$ is closed unbounded in $\omega_{1}$. If $\alpha$ happens to be a limit point of $C$, then the lemma is easy, so assume this does not happen. In this case, there is a maximal $\beta<\alpha$ that is closed under addition. The ordinal $\omega \cdot \beta$ is also closed under addition hence $\alpha \leq \omega \cdot \beta$. Since $\alpha$ is closed under addition, we must have $\alpha=\omega \cdot \beta$ and we are done if we set $\alpha_{n}=n \cdot \beta$.

Theorem 2. If $\alpha<\omega_{1}$ is closed under addition, then $\alpha$ is good.

Proof. The proof is by induction, with the case $\alpha=1$ already handled by Theorem 1 By Lemma 4.9, we can fix an increasing sequence $\left\{\alpha_{n}: n \in \omega\right\}$ cofinal in $\alpha$ such that for each $n$ (letting $\alpha_{-1}$ denote 0 ), the ordinal $\beta_{n}:=\operatorname{otp}\left(\alpha_{n} \backslash \alpha_{n-1}\right)$ is closed under addition.

For each $n$, we define

$$
\mathfrak{N}_{n}=\left\langle N_{i}: \alpha_{n-1}<i \leq \alpha_{n}\right\rangle .
$$

Note that $\mathfrak{N}_{n}$ is a relevant $\beta_{n}$-tower, and $\beta_{n}$ is good by the induction hypothesis.

For each $i$, there is a point $x_{i} \in \operatorname{Tr}\left(N_{\alpha_{i}}\right) \cap N_{\alpha_{i}+1}$ that witnesses that $\beta_{i}$ is good. There is an infinite $I \subseteq \omega$ such that the sequence $\left\{x_{i}: i \in I\right\}$ converges in $X$ to a point $\bar{x} \in \operatorname{Tr}\left(N_{\alpha}\right)$. We will show that this point $\bar{x}$ witnesses that $\alpha$ is good.

To do this, let $p \in N_{0} \cap P$ and let an open neighborhood $U$ of $\bar{x}$ be given. By throwing away finitely many members of $I$, we can assume $\left\{x_{i}: i \in I\right\} \subseteq U$. Let $\left\{B_{n}: n \in \omega\right\}$ be a decreasing neighborhood base for $\bar{x}$.

By induction on $n \in \omega$ we will produce objects $p_{n}, k(n), I_{n}$, and $U_{n}$ so that

(1) $p_{0}=p, I_{0}=I, U_{0}=U$,

(2) $k_{n}=\min \left(I_{n+1}\right)$,

(3) $p_{n+1} \leq p_{n}, k(n+1)>k(n), I_{n+1} \subseteq I_{n}, U_{n+1} \subseteq U_{n} \cap B_{n}$,

(4) $p_{n+1}$ is totally $\left(N_{\alpha_{k(n)}}, P\right)$-generic,

(5) $p_{n+1}$ is weakly $\left(\mathfrak{N}_{k(n)}, P\right)$-generic,

(6) $U_{n}$ is an open neighborhood of $\bar{x}$,

(7) $\left[p_{n+1}\right] \backslash\left[p_{n}\right] \subseteq U_{n+1}$,

(8) $I_{n}$ is an infinite subset of $\omega$,

(9) $\left\{x_{i}: i \in I_{n}\right\} \subseteq U_{n}$,

(10) for each promise $f$ appearing in $\Phi_{p_{m}}$ for some $m<\omega$, there is a stage $n$ such that

$$
\left\{x \in Y\left(f, p_{n}, p_{m}\right): U_{n+1} \subseteq f(x)\right\} \text { is large. }
$$

At a stage of the construction, we will have $p_{n}, I_{n}, U_{n}$, and a promise $f \in \Phi_{p_{i}}$ for some $i \leq n$ that we must take care of. The first thing we do is find $U_{n+1} \subseteq U_{n} \cap B_{n}$ such that

$$
\left\{x \in Y\left(f, p_{n}, p_{i}\right): U_{n+1} \subseteq f(x)\right\} \text { is large. }
$$


This is done just as in the proof of Theorem 1 Once this is done, we thin out $I_{n}$ to $I_{n+1}$ so that $\left\{x_{i}: i \in I_{n+1}\right\} \subseteq U_{n+1}$ and $k(n)>k(n-1)$. Now in the model $N_{\alpha_{k(n)}+1}$ we find a neighborhood $V$ of $x_{k(n)}$ with $V \subseteq U_{n+1}$. Finally, inside the model $N_{\alpha_{k(n)}+1}$ we apply our induction hypothesis to $p_{n}$ and the open set $V$ to obtain $p_{n+1}$. Now the verification that the sequence $\left\{p_{n}: n \in \omega\right\}$ has a lower bound proceeds just as in the proof of Theorem 1.

Corollary 4.10. $P$ is weakly $<\omega_{1}$-proper.

Proof. We prove this by induction on $\alpha<\omega_{1}$; the case where $\alpha=1$ is handled by Theorem [1]

Let $\mathfrak{N}=\left\langle N_{i}: i \leq \alpha\right\rangle$ be a relevant $\alpha$-tower for some $\alpha<\omega_{1}$, and let $p \in N_{0} \cap P$ be arbitrary.

If $\alpha$ is closed under addition, then Theorem 2 tells us there is a totally $\left(N_{\alpha}, P\right)$ generic $q \leq p$ that is weakly $(\mathfrak{N}, P)$-proper.

If $\alpha=\beta+\gamma$ for some strictly smaller $\beta$ and $\gamma$, then we apply the induction hypothesis for $\beta$ in the model $N_{\beta+1}$ to get a condition $q_{0} \leq p$ that is weakly $(\mathfrak{N} \uparrow \beta+1, P)$-generic, and then we apply the induction hypothesis for $\gamma$ to the tower $\mathfrak{N} \uparrow(\beta, \gamma]$ to get the required condition $q$.

\section{ITERATIONS OF WEAKLY $<\omega_{1}-$ PROPER FORCINGS}

We now prove a theorem that shows weak $<\omega_{1}$-properness is preserved by countable support iterations - this will be needed when we prove our main iteration theorem in the next section.

Before embarking on a proof, we set up our conventions regarding iterated forcing. For the most part, we follow the conventions of [19] and [12], though our notation differs in that we take " $q \leq p$ " to mean " $q$ extends $p$ ". We view conditions in the limit of a countable support iteration as functions with countable domains, so in particular we speak about domains of conditions as opposed to supports of conditions. We also assume that each partial order has a maximum element.

Theorem 3. Let $\mathbb{P}=\left\langle P_{\xi}, \dot{Q}_{\xi}: \xi<\kappa\right\rangle$ be a countable support iteration of forcings such that for each $\xi<\kappa$,

$$
\Vdash_{P_{\xi}} \dot{Q}_{\xi} \text { is weakly }<\omega_{1} \text {-proper. }
$$

If we are given objects $\alpha, \beta, \mathfrak{N}, \xi, \zeta$, and $p$ such that

(1) $\beta \leq \alpha<\omega_{1}$

(2) $\mathfrak{N}=\left\langle N_{i}: i \leq \alpha\right\rangle$ is a suitable tower,

(3) $\xi<\zeta$ in $N_{0} \cap(\kappa+1)$,

(4) $p \in N_{0} \cap P_{\zeta}$,

then there is a condition $r \in P_{\zeta}$ such that

(5) $\operatorname{dom} r \subseteq N_{\alpha} \cap[\xi, \zeta)$,

(6) whenever $q \in P_{\xi}$ satisfies

(a) $q \leq p\lceil\xi$,

(b) $q \Vdash \operatorname{Spec}\left(\mathfrak{N}, \dot{G}_{\xi}\right)$ is of order-type $\beta+1$, then

(c) $q \cup r \leq p$ in $P_{\zeta}$,

(d) $q \cup r \Vdash \operatorname{Spec}\left(\mathfrak{N}, \dot{G}_{\zeta}\right)$ is of order-type $\beta+1$. 
Proof. The proof requires lots of nested induction arguments, so we will take a little time and invest in notation that will make things a little easier.

For countable ordinals $\alpha$ and $\beta$, let $\circledast(\alpha, \beta)$ denote the statement:

$\circledast(\alpha, \beta)$ : Whenever $\mathfrak{N}=\left\langle N_{i}: i \leq \alpha\right\rangle$ is a suitable tower, $\xi<\zeta$ in $N_{0} \cap(\kappa+1)$, and $p \in N_{0} \cap P_{\zeta}$, then there is a condition $r \in P_{\zeta}$ such that $\operatorname{dom} r \subseteq N_{\alpha} \cap[\xi, \zeta)$, and whenever $q \in P_{\xi}$ satisfies

$$
q \leq p \nmid \xi
$$

and

$$
q \Vdash \operatorname{Spec}\left(\mathfrak{N}, \dot{G}_{\xi}\right) \text { is of order-type } \beta+1,
$$

then $q \cup r$ satisfies

$$
q \cup r \leq p
$$

and

$$
q \cup r \Vdash \operatorname{Spec}\left(\mathfrak{N}, \dot{G}_{\zeta}\right) \text { is of order-type } \beta+1 .
$$

We prove the statement $\circledast(\alpha, \beta)$ by induction on $\omega_{1} \times \omega_{1}$ with the lexicographic order. Note that if $\beta>\alpha$, then $\circledast(\alpha, \beta)$ holds trivially as there are no conditions $q$ satisfying (5.3)

Assume that the statement $\circledast\left(\alpha^{\prime}, \beta^{\prime}\right)$ holds for all pairs $\left(\alpha^{\prime}, \beta^{\prime}\right)<_{\text {lex }}(\alpha, \beta)$ and let $\mathfrak{N}, \xi, \zeta$, and $p$ be given.

The proof of the following claim is a standard "patching" argument. We include it for the convenience of readers who do not deal with iterated forcing on a daily basis.

Claim 5.1. Suppose $E \subseteq P_{\xi}$ is dense and open in the set of conditions that satisfy (5.2) and 5.3). If for each $u \in E$ there is an $r_{u} \in P_{\zeta}$ such that $\operatorname{dom} r_{u} \subseteq N_{\alpha} \cap[\xi, \zeta)$, $u \cup r_{u} \leq p$, and

$$
u \cup r_{u} \Vdash \operatorname{otp}\left(\operatorname{Spec}\left(\mathfrak{N}, \dot{G}_{\zeta}\right)\right)=\beta+1,
$$

then there is a single $r \in P_{\zeta}$ such that $\operatorname{dom} r \subseteq N_{\alpha} \cap[\xi, \zeta)$ and whenever $q \in P_{\xi}$ satisfies (5.2) and (5.3), $q \cup r$ satisfies (5.4) and (5.5).

Proof. Let $D$ be the set of conditions in $P_{\xi}$ that are either in $E$ are that have no extension in $E$. The set $D$ is dense and open in $P_{\xi}$, so we can find an antichain $\mathcal{A}$ maximal in $P_{\xi}$ that consists of elements of $D$. Let $\mathcal{A}_{0}=\mathcal{A} \cap E$. For each $u \in \mathcal{A}_{0}$, we let $r_{u}$ be the object given to us by our hypothesis, and let $s_{u}$ be $r_{u}$ augmented in the canonical way (each partial order has a maximum element!) so that dom $s_{u}=N_{\alpha} \cap[\xi, \zeta)$. For $u \in \mathcal{A} \backslash \mathcal{A}_{0}$, we let $s_{u}$ be any condition in $P_{\zeta}$ with domain $N_{\alpha} \cap[\xi, \zeta)$.

What follows now is a typical argument - we patch together all of the $s_{u}$ 's into a single object $r$ of the type we need. We define $r$ by induction on $N_{\alpha} \cap[\xi, \zeta)$. Given $\eta \in N_{\alpha} \cap[\xi, \zeta)$, we define

$$
r(\eta)=\left\{\left\langle u \cup\left(r\lceil\eta), s_{u}(\eta)\right\rangle: u \in \mathcal{A}\right\} .\right.
$$

Since $\mathcal{A}$ is a maximal antichain, it follows that $r(\eta)$ is a $P_{\eta}$-name for a condition in $\dot{Q}_{\eta}$. This defines $r$ and it follows from the construction that $r \in P_{\zeta}$ and $\operatorname{dom} r=$ $N_{\alpha} \cap[\xi, \zeta)$.

Now why does $r$ work? Note that if $q \in P_{\xi}$ satisfies (5.2) and (5.3), then $q$ forces the existence of a $u \in \mathcal{A}_{0}$ for which " $r=s_{u}$ ", and so (5.4) and (5.5) follow. 
Case $\beta$ is not closed under addition. . If $\beta$ is not closed under addition, then we can find ordinals $\gamma$ and $\gamma^{\prime}$ less than $\beta$ so that $\beta=\gamma+1+\gamma^{\prime}$. Note that we do not require that $\gamma$ and $\gamma^{\prime}$ are non-zero.

Definition 5.2. We define $E_{i}$ to be the set of all conditions $u \in P_{\xi}$ that extend $p\lceil\xi$ and that force each of the following statements to hold:

$$
\operatorname{otp}\left(\operatorname{Spec}\left(\mathfrak{N}, \dot{G}_{\xi}\right)\right)=\beta+1
$$

and

$$
i \text { is the } \gamma+1^{\text {st }} \text { member of } \operatorname{Spec}\left(\mathfrak{N}, \dot{G}_{\xi}\right) \text {. }
$$

We also define $E=\bigcup_{i<\alpha} E_{i}$.

Note that $E$ is dense below any condition in $P_{\xi}$ that satisfies (5.2) and (5.3) - we need only extend to decide a specific value $i$ for the $\gamma+1^{\text {st }}$ element of $\operatorname{Spec}\left(\mathfrak{N}, \dot{G}_{\xi}\right)$.

Claim 5.3. For each $i<\alpha$, there is a condition $r_{i} \in P_{\zeta}$ such that if $u \in E_{i}$, then

$$
u \cup r_{i} \leq p
$$

and

$$
u \cup r_{i} \Vdash \operatorname{otp}\left(\operatorname{Spec}\left(\mathfrak{N}, \dot{G}_{\zeta}\right)\right)=\beta+1 .
$$

Proof. We will obtain $r_{i}$ by applying our induction hypothesis twice. Inside the model $N_{i+1}$, we can apply our induction hypothesis to $\mathfrak{N}_{i}=\left\langle N_{j}: j \leq i\right\rangle$ to get a condition $s_{i} \in N_{i+1} \cap P_{\zeta}$ such that whenever $q \in P_{\xi}$ extends $p\lceil\xi$ and forces the order-type of $\operatorname{Spec}\left(\mathfrak{N}_{i}, \dot{G}_{\zeta}\right)$ to be $\gamma+1$, then

$$
q \cup s_{i} \leq p
$$

and

$$
q \cup s_{i} \Vdash \operatorname{otp}\left(\operatorname{Spec}\left(\mathfrak{N}_{i}, \dot{G}_{\zeta}\right)\right)=\gamma+1 .
$$

Let $\mathfrak{N}^{i}=\left\langle N_{j}: i<j \leq \alpha\right\rangle$. This tower is of length $\leq \alpha$, and since $\gamma^{\prime}<\beta$ we may apply our induction hypothesis to get a condition $r_{i} \in P_{\zeta}$ such that $\operatorname{dom} r_{i} \subseteq$ $N_{\alpha} \cap[\xi, \zeta)$ and whenever $q \in P_{\xi}$ forces $\operatorname{Spec}\left(\mathfrak{N}^{i}, \dot{G}_{\xi}\right)$ to be of order-type $\gamma^{\prime}+1$, we have

$$
q \cup r_{i} \leq s_{i}
$$

and

$$
q \cup r_{i} \Vdash \operatorname{otp}\left(\operatorname{Spec}\left(\mathfrak{N}^{i}, \dot{G}_{\zeta}\right)\right)=\gamma^{\prime}+1 .
$$

(Note that since $\xi \cap \operatorname{dom} s_{i}=\emptyset$, any condition in $P_{\xi}$ extends $s_{i}\lceil\xi$.)

Now suppose $u \in E_{i}$. Since $u$ forces (5.8) and (5.9) to hold and $\beta=\gamma+1+\gamma^{\prime}$, it must be the case that

$$
u \Vdash \operatorname{otp}\left(\operatorname{Spec}\left(\mathfrak{N}^{i}, \dot{G}_{\xi}\right)\right)=\gamma^{\prime}+1 .
$$

By our definition of $r_{i}$, we have that $u \cup r_{i} \leq s_{i}$ in $P_{\zeta}$ and

$$
u \cup r_{i} \Vdash \operatorname{otp}\left(\operatorname{Spec}\left(\mathfrak{N}^{i}, \dot{G}_{\zeta}\right)\right)=\gamma^{\prime}+1 .
$$

Taking this together with (5.9), we have that (5.11) holds as required. The proof is completed by a straightforward verification that $u \cup r_{i} \leq p$.

As commented earlier, the set $E$ is dense below any condition that forces (5.2) and (5.3) to hold, and so we may apply Claim 5.1 to get an object $r$ that will establish this particular instance of $\circledast(\alpha, \beta)$. 
Case $\beta$ is closed under addition. The proof of $\circledast(\alpha, \beta)$ in this case is by induction on the ordinal $\zeta$. Let $\left\langle\zeta_{n}: n<\omega\right\rangle$ be increasing and cofinal in $N_{\alpha} \cap \zeta$ with $\zeta_{0}=\xi$, and let $\left\langle\beta_{n}: n<\omega\right\rangle$ be increasing and cofinal in $\beta$.

Let us agree to call an element $s \in P_{\xi}$ relevant if $s \leq p\lceil\xi$ and $s$ forces the order-type of $\operatorname{Spec}\left(\mathfrak{N}, \dot{G}_{\xi}\right)$ to be $\beta+1$. If $s$ is relevant, then we say that $s$ can be completed if there is a $t \in P_{\zeta}$ such that $\operatorname{dom} t \subseteq N_{\alpha} \cap[\xi, \zeta), s \cup t \leq p$, and

$$
s \cup t \Vdash \operatorname{otp}\left(\operatorname{Spec}\left(\mathfrak{N}, \dot{G}_{\xi}\right)\right)=\beta+1 .
$$

Notice that the set of relevant conditions that can be completed is closed under extension, so it suffices to prove that every relevant condition has an extension that can be completed - if we show this, then a patching argument (as in Claim 5.1) will give us the object $r$ we need in order to finish.

Clearly every relevant condition can be extended to one that decides a particular value for the ordinal max $\operatorname{Spec}\left(\mathfrak{N}, \dot{G}_{\xi}\right)$, so what we will show is that any relevant condition that decides a value for $\max \operatorname{Spec}\left(\mathfrak{N}, \dot{G}_{\xi}\right)$ can be completed.

Note that if $s$ is relevant and $s \Vdash \max \operatorname{Spec}\left(\mathfrak{N}, \dot{G}_{\xi}\right)=\gamma+1 \leq \alpha$, then we are done by $\circledast(\gamma, \beta)$, so we need only worry about the other possibility.

Assume $s \in P_{\xi}$ satisfies

$$
\begin{gathered}
s \leq p\lceil\xi, \\
s \Vdash \operatorname{otp}\left(\operatorname{Spec}\left(\mathfrak{N}, \dot{G}_{\xi}\right)\right)=\beta+1,
\end{gathered}
$$

$$
s \Vdash \max \operatorname{Spec}\left(\mathfrak{N}, \dot{G}_{\xi}\right)=\alpha+1 .
$$

Our goal is to prove that $s$ can be completed; the first step in this task is a simple lemma about countable support iterations.

Lemma 5.4. If we are given objects $s_{n}$ and $\dot{p}_{n}($ for $n<\omega)$ such that

$$
\begin{aligned}
& \text { - } s_{n} \in P_{\zeta_{n}} \text {, } \\
& \text { - } p_{0} \in P_{\zeta} \text {, } \\
& \text { - } s_{0} \leq p_{0}\lceil\xi \text {, } \\
& \text { - } s_{n} \Vdash \dot{p}_{n+1} \in P_{\zeta} \text {, } \\
& \text { - } s_{n} \Vdash \dot{p}_{n+1}\left\lceil\zeta_{n} \in \dot{G}_{\zeta_{n}}\right. \text {, } \\
& \text { - } s_{n} \Vdash \dot{p}_{n+1} \leq \dot{p}_{n} \text {, } \\
& \text { - } s_{n+1}\left\lceil\zeta_{n}=s_{n}\right. \text {, } \\
& \text { then } \bigcup_{n<\omega} s_{n} \text { (a condition in } P_{\zeta} \text { ) forces that each } \dot{p}_{n} \text { is in } \dot{G}_{\zeta} \text {. }
\end{aligned}
$$

Proof. Let $G$ be any generic subset of $P_{\zeta}$ that contains $\bigcup_{n<\omega} s_{n}$; we will work in the model $V[G]$. Remember that $G \cap P_{\zeta_{n}}$ is a generic subset of $P_{\zeta_{n}}$ that contains $s_{n}$. For each $i<\omega$, let $p_{i+1}$ denote the interpretation of the name $\dot{p}_{i+1}$ using $G$ (or $G \cap P_{\zeta_{i}}$ if you prefer).

We need to show that $p_{i} \in G$ for each $i$. To do this, it suffices to prove that $p_{i}\left\lceil\zeta_{n} \in G\right.$ for each $n<\omega$. Why is this sufficient? For any $w \in P_{\zeta}$, the set of conditions in $P_{\zeta}$ that either extend $w$ or are incompatible with $w\left\lceil\zeta_{n}\right.$ for some $n$ is dense, so the genericity of $G$ comes to our rescue.

Since $s_{n} \in G$ for each $n$, we have that the sequence $\left\langle p_{i}: i<\omega\right\rangle$ is decreasing in $P_{\zeta}$. Now fix $n<\omega$. Since $s_{n} \in G$, we have that $p_{n}\left\lceil\zeta_{n} \in G\right.$, and since the sequence of $p_{i}$ 's is decreasing, this means that $p_{i}\left\lceil\zeta_{n} \in G\right.$ for $i<n$ as well. For $i>n$, we note that since $p_{i}\left\lceil\zeta_{i} \in G\right.$ we have $p_{i}\left\lceil\zeta_{n} \in G\right.$ as well. 
Now we return to the matter of showing that our given $s \in P_{\xi}$ can be completed. For $n \in \omega$, let $D_{n}$ be the set of conditions in $P_{\zeta}$ that force the order-type of $\operatorname{Spec}\left(\mathfrak{N}, \dot{G}_{\zeta}\right)$ to be greater than $\beta_{n}$.

By induction on $n<\omega$, we will define objects $\dot{p}_{n}, t_{n}$, and $s_{n}$ such that

(1) $\dot{p}_{0}=p, t_{0}=\emptyset, s_{0}=s$,

(2) $s_{n} \in P_{\zeta_{n}}$,

(3) $s_{n} \Vdash \dot{p}_{n+1} \in N_{\alpha} \cap P_{\zeta}$,

(4) $s_{n} \Vdash \dot{p}_{n+1} \uparrow \zeta_{n} \in \dot{G}_{\zeta_{n}}$,

(5) $t_{n} \in P_{\zeta}$,

(6) $\operatorname{dom} t_{n+1} \subseteq N_{\alpha} \cap\left[\zeta_{n}, \zeta_{n+1}\right)$,

(7) $s_{n+1}=s_{n} \cup t_{n+1}$,

(8) $s_{n} \Vdash \dot{p}_{n+1} \in D_{n}$,

(9) $s_{n} \Vdash \dot{p}_{n+1} \leq \dot{p}_{n}$.

Given $s_{n}, t_{n}$, and $\dot{p}_{n}$, we show how to obtain $\dot{p}_{n+1}, t_{n+1}$, and $s_{n+1}$. The next two lemmas will furnish us with $\dot{p}_{n+1}$.

Lemma 5.5. The set of conditions $u \in P_{\zeta_{n}}$ for which there exist $\bar{p} \in P_{\zeta}, \gamma_{0}$, and $\gamma_{1}$ such that

(1) $\gamma_{0}<\gamma_{1}<\alpha$,

(2) $\bar{p} \in N_{\gamma_{0}} \cap P_{\zeta}$,

(3) $u \Vdash \dot{p}_{n}=\bar{p}$,

(4) $u \Vdash \operatorname{otp}\left(\operatorname{Spec}\left(\mathfrak{N} \uparrow\left[\gamma_{0}, \gamma_{1}\right], \dot{G}_{\zeta}\right)\right)=\beta_{n}+1$

is dense below $s_{n}$.

Proof. Note that (5.21) together with our assumption that $\beta$ is closed under addition implies that $\alpha$ is a limit ordinal. Given an extension of $s$, we can extend it further to decide a particular value $\bar{p}$ for $\dot{p}_{n}$. Since $\alpha$ is a limit ordinal, this means there is some least $\gamma_{0}<\alpha$ for which $\bar{p} \in N_{\gamma_{0}}$. Since $\beta$ is closed under addition, it must be the case that our condition forces that the order-type of $\operatorname{Spec}\left(\mathfrak{N} \uparrow\left[\gamma_{0}, \alpha\right], \dot{G}_{\zeta}\right)$ is still $\beta+1$, and therefore we can extend again to decide a particular value $\gamma_{1}$ for the $\beta_{n}+1^{\text {st }}$ member of $\operatorname{Spec}\left(\mathfrak{N} \uparrow\left[\gamma_{0}, \alpha\right], \dot{G}_{\zeta}\right)$.

Lemma 5.6. Given $\gamma_{0}<\gamma_{1}<\alpha$ and $\bar{p} \in N_{\gamma_{0}} \cap P_{\zeta}$, there exist $v \in N_{\gamma_{1}+1}$ such that whenever $w \in P_{\zeta_{n}}$ extends $\bar{p} \uparrow \zeta_{n}$ and $w$ forces the order-type of $\operatorname{Spec}\left(\mathfrak{N} \uparrow\left[\gamma_{0}, \gamma_{1}\right], \dot{G}_{\zeta_{n}}\right)$ to be $\beta_{n}+1$, we have that $w \cup v \leq \bar{p}$ in $P_{\zeta}$ and $w \cup v$ forces the order-type of $\operatorname{Spec}\left(\mathfrak{N} \uparrow\left[\gamma_{0}, \gamma_{1}\right], \dot{G}_{\zeta}\right)$ to be $\beta_{n}+1$.

Proof. If we let $\alpha^{\prime}=\operatorname{otp}\left(\left[\gamma_{0}, \gamma_{1}\right]\right)$, then the lemma follows from our induction hypothesis $\circledast\left(\alpha^{\prime}, \beta_{n}\right)$.

Let $E_{n}$ be the set of all conditions $u \leq s_{n}$ in $P_{\zeta_{n}}$ that satisfy the assumptions of Lemma 5.5. For each $u \in E_{n}$, Lemma 5.6 provides us with an object $v_{u}$ such that

- $v_{u} \in N_{\alpha} \cap P_{\zeta}$,

- $\operatorname{dom} v_{u} \subseteq N_{\alpha} \cap\left[\zeta_{n}, \zeta\right)$,

- $u \cup v_{u} \Vdash \dot{p}_{n} \in \dot{G}_{\zeta}$,

- $u \cup v_{u} \Vdash \operatorname{otp}\left(\operatorname{Spec}\left(\mathfrak{N}, \dot{G}_{\zeta}\right)\right)>\beta_{n}$.

Let $\mathcal{A}_{n} \subseteq E_{n}$ be an antichain pre-dense below $s_{n}$, and define

$$
\dot{p}_{n+1}=\left\{\left\langle u, u \cup v_{u}\right\rangle: u \in \mathcal{A}_{n}\right\} .
$$


Now if $G$ is any generic subset of $P_{\zeta_{n}}$ that contains $s_{n}$, then there is a unique $u \in G \cap \mathcal{A}_{n}$. Since $\dot{p}_{n+1}$ gets interpreted as $u \cup v_{u}$, it follows easily that $s_{n}$ forces that $\dot{p}_{n+1}$ has all of the required properties.

To obtain $t_{n+1}$, we will do a similar argument in the poset $P_{\zeta_{n+1}}$. Note that if $u$ extends $s_{n}$ and there is some $\bar{p} \in P_{\zeta}$ such that $u \Vdash \dot{p}_{n+1}=\bar{p}$, then there is an object $v_{u}$ such that

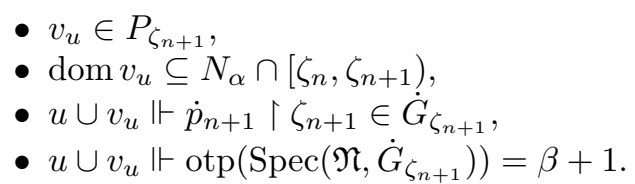

Such a $v_{u}$ can be found because of our induction hypothesis for $\zeta_{n+1}<\zeta$. A patching argument analogous to that of Claim 5.1 provides us with an object $t_{n+1}$ with the required properties. As $s_{n+1}$ is determined by $t_{n+1}$ and $s_{n}$, we have completed the construction for stage $n+1$.

Now we let $t=\bigcup_{n<\omega} t_{n}$. We need to show that $t$ completes $s$, i.e., that $\operatorname{dom} t \subseteq$ $N_{\alpha} \cap[\xi, \zeta), s \cup t \leq p$, and

$$
s \cup t \Vdash \operatorname{otp}\left(\operatorname{Spec}\left(\mathfrak{N}, \dot{G}_{\zeta}\right)\right)=\beta+1 .
$$

It is not hard to show that $\operatorname{dom} t \subseteq N_{\alpha} \cap[\xi, \zeta)$ and $s \cup t \leq p$ - both of these follow from the way $t$ was constructed. By Lemma 5.4 we have that $s \cup t$ forces that each $\dot{p}_{n}$ is in $G_{\zeta}$, and since $s \cup t$ extends $s_{n}$, we have that $\dot{p}_{n+1} \in D_{n}$ for each $n$. Thus

$$
s \cup t \Vdash \operatorname{otp}\left(\operatorname{Spec}\left(\mathfrak{N}, \dot{G}_{\zeta}\right)\right) \geq \sup _{n<\omega} \beta_{n}=\beta .
$$

Since $s \Vdash \operatorname{otp}\left(\operatorname{Spec}\left(\mathfrak{N}, \dot{G}_{\xi}\right)\right)=\beta+1$, it follows that

$$
s \cup t \Vdash \operatorname{otp}\left(\operatorname{Spec}\left(\mathfrak{N}, \dot{G}_{\zeta}\right)\right) \leq \beta+1 .
$$

Since $\operatorname{Spec}\left(\mathfrak{N}, \dot{G}_{\zeta}\right)$ is always a closed subset of $\alpha+1$ and $\beta$ is a limit ordinal, it must be the case that (5.23) holds.

To recap, what we have shown is that if $s$ is relevant and $s$ decides a specific value for the ordinal max $\operatorname{Spec}\left(\mathfrak{N}, \dot{G}_{\xi}\right)$, then $s$ can be completed. Since the set of such $s$ is dense in the set of all relevant conditions in $P_{\xi}$, we can use a patching argument to obtain a single $r$ that uniformly completes every relevant $s$. This shows us that our induction continues past stage $\zeta$, and from this we can deduce that $\circledast(\alpha, \beta)$ holds. This finishes the proof of Theorem 3 .

\section{ON ITERATIONS OF TOTALLY PROPER FORCINGS}

In this section, we show that weak $<\omega_{1}$-properness can serve as a replacement for $<\omega_{1}$-properness in the iteration theorems of [9] and Chapter V of [19] that deal with iterations that do not add reals. Shelah's paper [15] contains iteration theorems related to this one; he utilizes conditions even more technical than our requirement of weak $<\omega_{1}$-properness.

We will start with some notation germane to totally proper notions of forcing.

Definition 6.1. Let $P$ be a notion of forcing, let $N$ be a countable elementary submodel of some $H(\lambda)$ with $P \in N$, and let $p \in N \cap P$. We define

(1) $N^{P}=\{\dot{\tau} \in N: \dot{\tau}$ is a $P$-name $\}$,

(2) $\operatorname{Gen}(N, P)=\{G \subseteq N \cap P: G$ is an $N$-generic filter on $N \cap P\}$,

(3) $\operatorname{Gen}^{+}(N, P)=\{G \in \operatorname{Gen}(N, P): G$ has a lower bound in $P\}$, 
(4) $\operatorname{Gen}(N, P, p)=\{G \in \operatorname{Gen}(N, P): p \in G\}$,

(5) $\operatorname{Gen}^{+}(N, P, p)=\operatorname{Gen}(N, P, p) \cap \operatorname{Gen}^{+}(N, P)$.

Lemma 6.2. Assume $P$ is totally proper and $N$ is a countable elementary submodel of $H(\lambda)$ with $P \in N$.

(1) Each $G \in \operatorname{Gen}(N, P)$ is a maximal filter of conditions in $N \cap P$.

(2) If $p$ is totally $(N, P)$-generic, then $\{q \in N \cap P: p \leq q\}$ is in $\operatorname{Gen}(N, P)$.

(3) If $G_{0} \neq G_{1}$ in $\operatorname{Gen}(N, P)$, then there are $p_{i} \in G_{i}$ such that $p_{0}$ and $p_{1}$ are incompatible in $P$.

Proof. Left to the reader.

Definition 6.3. Let $P$ be a notion of forcing, let $A \subseteq P$, and let $\theta$ be a sentence of the forcing language. We define

$$
A \Vdash \theta
$$

to mean that whenever $G \subseteq P$ is a generic filter such that $A \subseteq G$, then $\theta$ holds in the generic extension $V[G]$.

If we adopt the convention that all notions of forcing are complete Boolean algebras, then " $A \Vdash \theta$ " is equivalent to the condition $\wedge A$ (the infimum of $A$ ) forcing $\theta$ to be true.

Proposition 6.4. Let $P$ be a totally proper notion of forcing, and let $N$ be a countable elementary submodel of $H(\lambda)$ that contains $P$. Let $\dot{\tau}_{0}, \ldots, \dot{\tau}_{n-1}$ be elements of $N^{P}$, and let $\theta\left(x_{0}, \ldots, x_{n-1}\right)$ be a formula. Let $H \subseteq P$ be a filter of conditions that satisfies $N \cap H \in \operatorname{Gen}(N, P)$. Then either

$$
H \Vdash \theta\left(\dot{\tau}_{0}, \ldots, \dot{\tau}_{n-1}\right),
$$

or

$$
H \Vdash \neg \theta\left(\dot{\tau}_{0}, \ldots, \dot{\tau}_{n-1}\right) .
$$

Proof. This follows because elements of $\operatorname{Gen}(N, P)$ are maximal filters on the countable set $N \cap P$.

Lemma 6.5. Let $P$ be totally proper, and let $N_{0} \in N_{1}$ be countable elementary submodels of $H(\lambda)$. Suppose that $\bar{G} \in \operatorname{Gen}\left(N_{0}, P\right), G \in \operatorname{Gen}^{+}\left(N_{1}, P\right)$, and $\bar{G} \subseteq G$. Then $\bar{G} \in N_{1}$.

Proof. Let $D$ be the set of conditions in $P$ that are either totally $\left(N_{0}, P\right)$-generic, or that have no totally $\left(N_{0}, P\right)$-generic extension. Clearly $D$ is dense in $P$, and $D \in N_{1}$. Thus there is a $p \in N_{1} \cap G$. Let $r$ be a lower bound for $G$. Then $r \leq p$ and $r$ is a lower bound for $\bar{G}$ as well, and therefore $p$ has a totally $\left(N_{0}, P\right)$-generic extension. Since $p \in D$, it must be the case that $p$ is totally $\left(N_{0}, P\right)$-generic. By part (2) of Lemma 6.2 $G^{\prime}=\left\{q \in N_{0} \cap P: p \leq q\right\}$ is in $\operatorname{Gen}\left(N_{0}, P\right)$, and furthermore $G^{\prime} \in N_{1}$ as it is definable from $N_{0}, p$, and $P$. Since $G$ is a filter, every member of $\bar{G}$ is compatible with $p$, and hence by part (3) of Lemma 6.2, it must be the case that $G^{\prime}=G$, and thus $G \in N_{1}$.

The proof of the next lemma is left to the reader - it follows easily from the definitions involved. 
Lemma 6.6. Let $P$ be totally proper, and suppose $\dot{Q}$ is a $P$-name for a totally proper notion of forcing. Let $N \prec H(\lambda)$ be countable, with $\{P, \dot{Q}\} \in N$. Let $G \in \operatorname{Gen}(N, P)$, as suppose $\left\{\dot{q}_{n}: n \in \omega\right\}$ is an $(N[G], \dot{Q})$-generic sequence. Then $H:=\left\{r \in N \cap P * \dot{Q}:(\exists p \in G)(\exists n \in \omega)\left[p * \dot{q}_{n} \leq r\right]\right\}$ is an element of $\operatorname{Gen}(N, P * \dot{Q})$.

The reader should contrast the result of the next lemma with the definition of weak $<\omega_{1}$-properness; here we find a condition $q$ that explicitly determines the members of the spectrum of genericity.

Lemma 6.7. Suppose $P$ is totally proper and weakly $<\omega_{1}$-proper. Let $\mathfrak{N}=\left\langle N_{i}\right.$ : $i \leq \alpha\rangle$ be a suitable tower of models. Given $p \in N \cap P$, there is a $q \leq p$ such that $\left\{i \leq \alpha: q\right.$ is totally $\left(N_{i}, P\right)$-generic $\}$ has order-type $\alpha+1$.

Proof. Since $P$ is weakly $<\omega_{1}-$ proper, there is an $r \leq p$ such that

$$
r \Vdash \operatorname{Spec}(\mathfrak{N}, P) \text { has order-type } \alpha+1 \text {. }
$$

Since $P$ is totally proper and $\alpha$ is countable, there is a $q \leq r$ and a set $I \subseteq \alpha+1$ such that

$$
q \Vdash I=\operatorname{Spec}(\mathfrak{N}, P) .
$$

Clearly $I$ must have order-type $\alpha+1$, and for $i \in I$,

$$
q \Vdash N_{i}[\dot{G}] \cap \operatorname{Ord}=N_{i} \cap \text { Ord, }
$$

hence $q$ is $\left(N_{i}, P\right)$-generic.

Note that in the conclusion of the preceding lemma, the set of $i \leq \alpha$ such that $q$ is totally $\left(N_{i}, P\right)$-generic must be a suitable index set - this follows from Proposition 4.5 .

The next definition appears implicitly in [19] and it is intimately related to ${ }^{\omega} \omega_{-}$ bounding notions of forcing. The notation " $(N, P)^{\text {fin }}$-generic" is new although the property so defined has played a prominent role in Shelah's work - our choice of notation seems like a convenient way of expressing the concepts involved.

Definition 6.8. Let $P$ be a notion of forcing and let $N \prec H(\lambda)$ be countable with $P \in N$. A condition $q \in P$ is said to be $(N, P)^{\text {fin }}$-generic if for every $D \in N$ dense in $P$, there is a finite subset of $N \cap D$ that is pre-dense below $p$.

It is instructive to compare the preceding definition to the definitions of " $q$ is $(N, P)$-generic" and " $q$ is totally $(N, P)$-generic". The best way to see what is going on is to look at maximal antichains $\mathcal{A} \subseteq P$ that are elements of $N$. If $q$ is $(N, P)$-generic, then $q$ forces $G \cap \mathcal{A} \in N$. If $q$ is $(N, P)^{\text {fin }}$-generic, then in addition there is a finite subset $\mathcal{A}_{0}$ of $N \cap \mathcal{A}$ such that $p \Vdash G \cap \mathcal{A} \in \mathcal{A}_{0}$. If $q$ is totally $(N, P)$-generic, then there is some unique condition $q \in N \cap \mathcal{A}$ with $p \leq q$.

We previously mentioned ${ }^{\omega} \omega$-bounding notions of forcing, so we take a moment to recall the definition (from [19]) and pin down how it relates to $(N, P)^{\mathrm{fin}}$ genericity.

Definition 6.9. A forcing notion $P$ is ${ }^{\omega} \omega$-bounding if for every $f \in{ }^{\omega} \omega^{V[G]}$ there is a $g \in \omega^{\omega} \omega^{V}$ such that for all $n \in \omega, f(n) \leq g(n)$.

The following result has a right to be called folklore - the authors are not aware if it has explicitly appeared in the literature, but it has certainly been known since very early on in the history of proper forcing. 
Proposition 6.10. A notion of forcing $P$ is proper and ${ }^{\omega} \omega$-bounding if and only if whenever $N \prec H(\lambda)$ is countable with $P \in N$, and $p \in N \cap P$, there is a $q \leq p$ such that $q$ is $(N, P)^{\text {fin }}$-generic.

Proof. Suppose $P$ is proper and ${ }^{\omega} \omega$-bounding, and let $N \prec H(\lambda)$ be a countable model containing $P$. Given $p \in N \cap P$, let $r \leq q$ be $(N, P)$-generic. Next, choose $N_{1} \prec H(\lambda)$ countable such that $N$ and $r$ are in $N_{1}$. Inside $N_{1}$, let $\left\langle\mathcal{A}_{n}: n \in \omega\right\rangle$ list all maximal antichains of $P$ that are elements of $N$, and for each $n$, let $\left\langle a_{n}^{i}: i<\omega\right\rangle$ enumerate $N \cap \mathcal{A}_{n}$.

Since $r$ is $(N, P)$-generic, there is a $P$-name $\dot{f}$ for a function in ${ }^{\omega} \omega$ such that

$$
r \Vdash(\forall n<\omega)\left[N \cap \dot{G} \in\left\{a_{n}^{i}: i \leq \dot{f}(n)\right\}\right] .
$$

Since $P$ is ${ }^{\omega} \omega$-bounding, there is a $q \leq r$ and $g \in{ }^{\omega} \omega$ such that for each $n, q$ forces that $\dot{f}(n) \leq g(n)$. One can then easily check that $q$ is $(N, P)^{\text {fin }}$-generic as required.

For the converse, let $\dot{f}$ be a $P$-name for a function in ${ }^{\omega} \omega$. Let $N$ be a countable elementary submodel of $H(\lambda)$ that contains $P$ and $\dot{f}$, and let $p \in N \cap P$ be arbitrary. There is a $q \leq p$ such that $q$ is $(N, P)^{\text {fin }}$-generic. For each $n$, the set of conditions that decide a value for $\dot{f}(n)$ is dense in $P$ and an element of $N$. Since $q$ is $(N, P)^{\text {fin }}$ generic, for each $n$ there is some $g(n) \leq \omega$ such that $q \Vdash \dot{f}(n) \in g(n)$, and we are done.

If $P * \dot{Q}$ is a two step iteration, $p$ is $(N, P)^{\text {fin }}{ }_{\text {-generic and }}$

$$
p \Vdash \text { " } \dot{q} \text { is }(N[\dot{G}], \dot{Q})^{\text {fin }} \text {-generic", }
$$

then in general we cannot conclude that $p * \dot{q}$ is $(N, P * \dot{Q})^{\text {fin }}$-generic. The following lemma, taken from Chapter XVIII of [19], tells us that in certain cases we can remedy this shortcoming; it will play a crucial role in the proof of Theorem 4 .

Lemma 6.11 (Claim XVIII.2.6 of [19]). Let $\mathbb{P}=\left\langle P_{i}, \dot{Q}_{i}: i\langle\kappa\rangle\right.$ be a countable support iteration of ${ }^{\omega} \omega$-bounding proper forcing notions. Given objects $i, j, N_{0}$, $N_{1}, p$, and $q$ such that

(1) $i<j \leq \kappa$,

(2) $N_{0} \in N_{1}$ are countable elementary submodels of $H(\lambda)$ such that $\{\mathbb{P}, i, j\} \in$ $N_{0}$,

(3) $p \in N_{0} \cap P_{j}$,

(4) $q \in P_{i}$,

(5) $q \leq p\lceil i$,

(6) $q$ is $\left(N_{0}, P_{i}\right)^{\mathrm{fin}}$-generic,

(7) $q$ is $\left(N_{1}, P_{i}\right)$-generic,

there is an object $r$ such that

(8) $r \in P_{j}$,

(9) $r\lceil i=q$,

(10) $r \leq p$,

(11) $r$ is $\left(N_{0}, P_{j}\right)^{\text {fin }}$-generic,

(12) $r$ is $\left(N_{1}, P_{j}\right)$-generic.

The preceding lemma is an essential ingredient in the proof of the upcoming iteration theorem. Although it is not explicitly stated in the conclusion of the lemma, one can obtain that $\operatorname{dom} r \subseteq \operatorname{dom} q \cup N_{1} \cap[i, j)$. 
The next theorem is modelled on the proofs found in Chapter VIII of [19]. Conceptually, our proof is no more difficult than those in Shelah's book - the arguments are at the heart the same - but things are a lot more complex at the technical level in our proof because weak $<\omega_{1}$-properness is not as nicely behaved as honest-to-goodness $<\omega_{1}$-properness.

Theorem 4. Let $\mathbb{P}=\left\langle P_{\alpha}, \dot{Q}_{\alpha}: \alpha<\epsilon\right\rangle$ be a countable support iteration such that:

(1) $\Vdash_{P_{\alpha}} \dot{Q}_{\alpha}$ is totally proper.

(2) $\Vdash_{P_{\alpha}} \dot{Q}_{\alpha}$ is weakly $<\omega_{1}$-proper.

(3) For each $\alpha$, if $N_{0}, N_{1}, \dot{q}, \bar{G}$, and $\left\langle G^{\ell}: \ell<k\right\rangle$ satisfy

- $N_{0}$ and $N_{1}$ are countable elementary submodels of $H(\lambda)$,

- $N_{0} \in N_{1}$,

- $\{\mathbb{P}, \alpha, \dot{q}\} \in N_{0}$,

- $\Vdash_{P_{\alpha}} \dot{q} \in \dot{Q}_{\alpha}$,

- $\bar{G} \in \mathrm{Gen}^{+}\left(N_{0}, P_{\alpha}, p\right) \cap N_{1}$,

- for $\ell<k, G^{\ell} \in \operatorname{Gen}\left(N_{1}, P_{\alpha}\right)$,

- for $\ell<k, \bar{G} \subseteq G^{\ell}$,

then there is a sequence $\left\langle\dot{q}_{n}: n \in \omega\right\rangle$ in $N_{1} \cap \operatorname{Gen}\left(N_{0}[\bar{G}], \dot{Q}, \dot{q}\right)$ such that for all $\ell<k$,

$$
G^{\ell} \Vdash\left\langle\dot{q}_{n}: n \in \omega\right\rangle \text { has a lower bound in } \dot{Q} \text {. }
$$

Then $P_{\epsilon}$ is totally proper.

Before we get into a proof, let us comment that condition (3) of the statement of the theorem is a weakened version of the condition used in the iteration theorem presented in 9], and all iteration theorems in the literature that involve not adding reals require something along these lines — in Shelah's terminology, this is "medicine against the weak diamond" (see [15]).

Let $N_{0} \prec H(\lambda)$ be a countable model such that $\mathbb{P} \in N_{0}$.

Definition 6.12. For $\xi<\zeta \in N_{0} \cap(\epsilon+1)$, we define an ordinal $\alpha(\xi, \zeta)$ as follows: Given $\zeta$, we let

$$
\alpha=\sup \left\{\alpha\left(\xi^{\prime}, \zeta^{\prime}\right):\left(\xi^{\prime}, \zeta^{\prime}\right) \in N_{0} \text { and either } \zeta^{\prime}<\zeta \text { or } \zeta^{\prime}=\zeta \wedge \xi^{\prime}<\xi\right\},
$$

and let $\alpha(\xi, \zeta)=\alpha_{0}+3$, where $\alpha_{0}$ is the least ordinal greater than $\alpha$ that is closed under ordinal addition.

At this point, the previous definition may seem to be a bit mysterious. What's going on is that $\alpha(\xi, \zeta)$ is going to be the length of a tower of models that is "long enough" to allow us to use an induction hypothesis to advance from $P_{\xi}$ to $P_{\zeta}$ in the proof of the iteration theorem.

Proposition 6.13. Given $\xi<\zeta \in N_{0} \cap(\epsilon+1), p, \mathfrak{N}, \bar{G},\left\langle G^{\ell}: \ell<k\right\rangle$, and $\left\langle r^{\ell}: \ell<k\right\rangle$ such that

(1) $p \in N_{0} \cap P_{\zeta}$,

(2) $\mathfrak{N}=\left\langle N_{\beta}: \beta \leq \alpha(\xi, \zeta)\right\rangle$ satisfies

- $\mathfrak{N}$ is a continuous increasing $\in$-chain of countable elementary submodels of $H(\lambda)$,

- $\left\langle N_{\beta}: \beta \leq \alpha\right\rangle \in N_{\alpha+1}$,

(3) $\bar{G} \in \operatorname{Gen}^{+}\left(N_{0}, P_{\xi}\right) \cap N_{1}$,

(4) $p \uparrow \xi \in \bar{G}$, 
(5) $\left\langle G^{\ell}: \ell<k\right\rangle$ satisfies

- $G^{\ell} \in \mathrm{Gen}^{+}\left(N_{\alpha(\xi, \zeta)}, P_{\xi}\right)$,

- $\bar{G} \subseteq G^{\ell}$,

- $\left\{\beta \leq \alpha(\xi, \zeta):(\forall \ell<k)\left[N_{\beta} \cap G^{\ell} \in \operatorname{Gen}\left(N_{\beta}, P_{\xi}\right)\right]\right\}$ has order-type $\alpha(\xi, \zeta)$,

(6) $r^{\ell}$ is a lower bound for $G^{\ell}$,

then we can find objects $G^{*}$ and s such that

(1) $G^{*} \in \operatorname{Gen}\left(N_{0}, P_{\zeta}\right)$,

(2) $p \in G^{*}$,

(3) $s \in P_{\zeta}$ with $\operatorname{dom} s \subseteq N_{\alpha(\xi, \zeta)} \cap[\xi, \zeta)$,

(4) for each $\ell<k, r^{\ell} \cup s$ is a lower bound for $G^{*}$ in $P_{\zeta}$.

Proof. The proof is by induction on $\zeta \in N_{0} \cap(\epsilon+1)$.

CASE 1: $\zeta=\zeta_{0}+1$.

Here, the way is clear - we will use our induction hypothesis to move things to $P_{\zeta_{0}}$, and then use condition (3) of Theorem 4 to advance one more step.

Let $\xi<\zeta$ in $N_{0} \cap(\epsilon+1)$. The case where $\xi=\zeta_{0}$ follows immediately from our assumptions, so assume that $\xi<\zeta_{0}$.

Let $p, \mathfrak{N}, \bar{G},\left\langle G^{\ell}: \ell<k\right\rangle$, and $\left\langle r^{\ell}: \ell<k\right\rangle$ be given. Let $\alpha=\alpha\left(\xi, \zeta_{0}\right)$. By Lemma 6.5. we know that for each $\ell<k, N_{\alpha} \cap G^{\ell} \in N_{\alpha+1}$ and so for each $\ell<k$ we can find a condition $r_{\ell} \in N_{\alpha+1} \cap G^{\ell}$ that is a lower bound for $N_{\alpha} \cap G^{\ell}$.

Now inside $N_{\alpha+1}$ we apply our induction hypothesis to the objects $p\left\lceil\zeta_{0}, \bar{G}\right.$, $\left\langle N_{\alpha} \cap G^{\ell}: \ell<k\right\rangle$, and $\left\langle r_{\ell}: \ell<k\right\rangle$.

This gives us objects $H^{*}$ and $t$ such that

- $H^{*} \in \operatorname{Gen}\left(N_{0}, P_{\zeta_{0}}, p\left\lceil\zeta_{0}\right) \cap N_{\alpha+1}\right.$,

- $r_{\ell} \cup t$ is a lower bound for $H^{*}$ for each $\ell<k$.

Now in the model $N_{\alpha+2}$, we can find conditions $t^{\ell} \in P_{\zeta_{0}}$ such that $t^{\ell} \uparrow \xi \in G^{\ell}$, $t^{\ell} \leq r_{\ell} \cup t$, and $t^{\ell}$ is totally $\left(N_{\alpha+1}, P_{\zeta_{0}}\right)$-generic, say

$$
t^{\ell} \Vdash N_{\alpha+1} \cap \dot{G}_{\zeta_{0}}=H^{\ell} .
$$

Now we apply assumption (3) of Theorem 4 to the objects $N_{0} \in N_{\alpha+1}, p(\zeta), H^{*}$, $\left\langle H^{\ell}: \ell<k\right\rangle$ to get an $\left(N_{0}\left[H^{*}\right], \dot{Q}_{\zeta}, p(\zeta)\right)$-generic sequence $\left\langle\dot{q}_{n}: n<\omega\right\rangle$ in $N_{\alpha+1}$ that each $H^{\ell}$ forces to have a lower bound. Now that we have done this, Lemma 6.6 shows us how to obtain $G^{*} \in \operatorname{Gen}\left(N_{0}, P_{\zeta}\right)$ from $H^{*}$ and the sequence $\left\{\dot{q}_{n}: n \in \omega\right\}$.

CASE 2: $\zeta$ a limit.

Let $\left\{D_{n}: n \in \omega\right\}$ list the dense open subsets of $P_{\zeta}$ that are elements of $N_{0}$, and let $\left\langle\zeta_{n}: n \in \omega\right\rangle$ be cofinal in $N_{0} \cap \zeta$ with $\zeta_{0}=\xi$. Also, let $\alpha_{0}$ be the unique ordinal such that $\alpha(\xi, \zeta)=\alpha_{0}+3$. Recall that $\alpha_{0}$ is closed under ordinal addition.

By induction, we will define objects $p_{n}, G_{n}^{*}, I_{n}, k_{n},\left\langle G_{n}^{\bar{\ell}}: \bar{\ell}<k_{n}\right\rangle$, and $s_{n}$ such that

(1) $p_{0}=p, G_{0}^{*}=\bar{G}, I_{0}=\alpha_{0}+1, k_{0}=k, G_{0}^{\ell}=G^{\ell} \cap N_{\alpha_{0}+1}, s_{0}=\emptyset$,

(2) $p_{n+1} \leq p_{n}$

(3) $p_{n+1} \in N_{0} \cap D_{n}$,

(4) $p_{n+1}\left\lceil\zeta_{n} \in G_{n}^{*}\right.$,

(5) $G_{n}^{*} \in \operatorname{Gen}^{+}\left(N_{0}, P_{\zeta_{n}}\right) \cap N_{\min \left(I_{n} \backslash\{0\}\right)}$,

(6) $G_{n+1}^{*} \cap P_{\zeta_{n}}=G_{n}^{*}$,

(7) $G_{n}^{\bar{\ell}} \in \operatorname{Gen}^{+}\left(N_{\alpha_{0}+1}, P_{\zeta_{n}}\right) \cap N_{\alpha_{0}+2}$,

(8) $G_{n}^{*} \subseteq G_{n}^{\bar{\ell}}$ for all $\bar{\ell}<k_{n}$, 
(9) $I_{n}=\left\{\beta \leq \alpha_{0}: N_{\beta} \cap G_{n}^{\bar{\ell}} \in \operatorname{Gen}\left(N_{\beta}, P_{\zeta_{n}}\right)\right.$ for all $\left.\bar{\ell}<k_{n}\right\}$,

(10) the order-type of $I_{n}$ is $\alpha_{0}+1$,

(11) $s_{n} \in P_{\zeta_{n}}$ with domain a subset of $\left[\xi, \zeta_{n}\right)$,

(12) for each $\ell<k$, we have

- $r^{\ell} \cup s_{n} \in P_{\zeta_{n}}$,

- $r^{\ell} \cup s_{n} \Vdash N_{\alpha_{0}+1} \cap \dot{G}_{\zeta_{n}} \in\left\{G_{n}^{\bar{\ell}}: \bar{\ell}<k_{n}\right\}$,

- $r^{\ell} \cup s$ is $\left(N_{\alpha_{0}+2}, P_{\zeta_{n}}\right)^{\text {fin }}$-generic,

- $r^{\ell} \cup s_{n}$ is $\left(N_{\alpha_{0}+3}, P_{\zeta_{n}}\right)$-generic.

Given the objects for stage $n$, we must show how to get the objects required for stage $n+1$. Throughout the upcoming argument, we will be exchanging "vertical information" for "horizontal information". To understand what this means, the reader should look at how well the conditions $r^{\ell} \cup s_{n}$ "pin down" the identity of the generic object $\dot{G}_{\zeta_{n}}$.

For example, all of the conditions $r^{\ell} \cup s_{n}$ are $\left(N_{\alpha_{0}+3}, P_{\zeta_{n}}\right)$-generic. This means that given a maximal antichain $\mathcal{A}$ of $P_{\zeta_{n}}$ that lies in $N_{\alpha_{0}+3}, r^{\ell} \cup s_{n}$ will guarantee that the generic object hits the countable set $\mathcal{A} \cap N_{\alpha_{0}+3}$. If $\mathcal{A}$ happens to be in $N_{\alpha_{0}+2}$, then $r^{\ell} \cup s_{n}$ can see a finite subset of $N_{\alpha_{0}+2} \cap \mathcal{A}$ such that it, i.e., $r^{\ell} \cup s_{n}$, can guarantee the generic object will meet that finite set.

As far as $N_{\alpha_{0}+1}$ is concerned, we are much better off - we actually have a finite list of candidates $\left\langle G_{n}^{\bar{\ell}}: \bar{\ell}<k_{n}\right\rangle$ such that for each $\bar{\ell}<k$,

$$
r^{\ell} \cup s_{n} \Vdash\left(\exists \bar{\ell}<k_{n}\right)\left[N_{\alpha_{0}+1} \cap \dot{G}_{\zeta_{n}}\right]=G_{n}^{\bar{\ell}} .
$$

Next, consider how things improve when we move to the model $N_{\alpha_{0}}$. Not only does $r^{\ell} \cup s_{n}$ guarantee that the generic object looks like a restriction of one of the $G_{n}^{\bar{\ell}}$ 's to $N_{\alpha_{0}}$, we also have that the sets $G_{n}^{\bar{\ell}}$ for $\bar{\ell}<k_{n}$ are simultaneously generic for lots of the models $N_{\beta}, \beta<\alpha_{0}$.

Finally, each $r^{\ell} \cup s_{n}$ is totally $\left(N_{0}, P_{\zeta_{n}}\right)$-generic because they all agree that the generic object, when restricted to the model $N_{0}$, looks like $G_{n}^{*}$. One should think of this as "the degree of genericity of $r^{\ell} \cup s_{n}$ decreases as we move up through the tower".

Now we get back to the task of building the objects needed for stage $n+1$.

Goal: Find $p_{n+1}$.

Let us define

$$
D=\left\{q \in P_{\zeta_{n}}:\left(\exists p \in D_{n}\right)\left[p \in D_{n} \text { extends } p_{n} \text { and } p\left\lceil\zeta_{n}=q\right]\right\} .\right.
$$

It is clear that $D$ is dense below $p_{n} \uparrow \zeta_{n}$ in $P_{\zeta_{n}}$. Since $G_{n}^{*} \in \operatorname{Gen}\left(N_{0}, P_{\zeta_{n}}\right)$ and $p_{n} \uparrow \zeta_{n} \in G_{n}^{*}$, we can find the required $p_{n+1}$ using the genericity of $G_{n}^{*}$.

Goal: Find $G_{n+1}^{*}$.

Let $\beta_{0} \in I_{n}$ be such that $\operatorname{otp}\left(I_{n} \cap \beta_{0}\right)=\alpha\left(\zeta_{n}, \zeta_{n+1}\right)$, and we let $\beta_{1}$ equal the next element of $I_{n}$ past $\beta_{0}$. Inside $N_{\beta_{1}}$, for each $\bar{\ell}<k_{n}$ we choose $r_{\beta_{1}}^{\bar{\ell}} \in G_{n}^{\bar{\ell}}$ that is a lower bound for $N_{\beta_{0}} \cap G_{n}^{\bar{\ell}}$, and let $H^{\bar{\ell}} \in \operatorname{Gen}\left(N_{\beta_{0}}, P_{\zeta_{n}}\right)$ be such that $r_{\beta_{1}}^{\bar{\ell}} \Vdash N_{\beta_{0}} \cap G_{n}^{\bar{\ell}}=H^{\bar{\ell}}$.

We apply our induction hypothesis for $P_{\zeta_{n+1}}$ inside the model $N_{\beta_{1}}$ to the objects

- $p \nmid \zeta_{n+1}$,

- $\left\langle N_{\beta}: \beta \in I_{n} \cap\left(\beta_{0}+1\right)\right\rangle$,

- $G_{n}^{*}$

- $\left\langle H^{\bar{\ell}}: \bar{\ell}<k_{n}\right\rangle$,

- $\left\langle r_{\beta_{1}}^{\bar{\ell}}: \bar{\ell}<k_{n}\right\rangle$, 
and this gives us $G_{n+1}^{*} \in N_{\beta_{1}} \cap \operatorname{Gen}\left(N_{0}, P_{\zeta_{n+1}}\right)$ and $t \in N_{\beta_{1}}$ such that $p_{n+1}\left\lceil\zeta_{n+1} \in\right.$ $G_{n+1}^{*}$, and for all $\bar{\ell}<k_{n}, r_{\beta_{1}}^{\bar{\ell}} \cup t$ is a lower bound for $G_{n+1}^{*}$. Note that Proposition 4.5 tells us that $\left\langle N_{\beta}: \beta \in I_{n} \cap \beta_{0}+1\right\rangle$ is as needed in order to apply our induction hypothesis.

Goal: Find $I_{n+1}$.

We now work inside the model $N_{\alpha_{0}+1}$. For $\bar{\ell}<k_{n}$, choose $r_{\alpha_{0}}^{\bar{\ell}} \in N_{\alpha_{0}+1} \cap G_{n}^{\bar{\ell}}$ that is a lower bound for $N_{\alpha_{0}} \cap G_{n}^{\bar{\ell}}$. Let $J_{0}=I_{n} \backslash\left(\beta_{1}+1\right)$.

By induction on $\bar{\ell}<k_{n}$ we will define objects $t_{\bar{\ell}}$ and $J_{\bar{\ell}+1}$ such that

- $t_{\bar{\ell}} \leq r_{\alpha_{0}}^{\bar{\ell}} \cup t$ in $P_{\zeta_{n+1}}$,

- $t_{\bar{\ell}}$ is totally $\left(N_{\alpha_{0}}, P_{\zeta_{n+1}}\right)$-generic,

- $t_{\bar{\ell}}\left\lceil\zeta_{n} \in N_{\alpha_{0}+1} \cap G_{n}^{\bar{\ell}}\right.$,

- $t_{\bar{\ell}}$ is weakly $\left(\mathfrak{N} \uparrow J_{\bar{\ell}}, P_{\zeta_{n+1}}\right)$-generic,

- $J_{\bar{\ell}+1}:=\left\{\beta \in J_{\bar{\ell}}: t_{\bar{\ell}}\right.$ is totally $\left(N_{\beta}, P_{\zeta_{n+1}}\right)$-generic $\}$ has order-type $\alpha_{0}+1$.

Note that $J_{0}$ is a suitable index set and $J_{0} \in N_{\alpha_{0}+1}$. Given $J_{\bar{\ell}}$, we show how to obtain $t_{\bar{\ell}}$ and $J_{\bar{\ell}+1}$.

Our assumptions on $J_{\bar{\ell}}$ imply that $\mathfrak{N} \uparrow J_{\bar{\ell}}$ is a suitable tower of models and $\mathfrak{N}\left\lceil J_{\bar{\ell}} \in N_{\alpha_{0}+1}\right.$. Also, since $J_{\bar{\ell}}$ is a subset of $I_{n}$, we know that $r_{\alpha_{0}}^{\bar{\ell}}$ is weakly $\left(\mathfrak{N} \uparrow J_{\bar{\ell}}, P_{\zeta_{n}}\right)$-generic.

We now apply Theorem 3 inside the model $N_{\alpha_{0}+1}$ for the partial order $P_{\zeta_{n+1}}$ with $\mathfrak{N} \uparrow J_{\bar{\ell}}, r_{\alpha_{0}}^{\bar{\ell}}$, and $r_{\beta_{1}}^{\bar{\ell}} \cup t$ in place of the $\mathfrak{N}$, $q$, and $p$ appearing there. This gives us an object $s^{\bar{\ell}}$ that "lengthens" $r_{\alpha_{0}}^{\bar{\ell}}$ into a weakly $\left(\mathfrak{N} \uparrow J_{\bar{\ell}}, P_{\zeta_{n+1}}\right)$-generic condition.

Claim 6.14. There is a totally $\left(N_{\alpha_{0}}, P_{\zeta_{n+1}}\right)$-generic condition $t^{\bar{\ell}}$ in $N_{\alpha_{0}+1}$ that extends $r_{\alpha_{0}}^{\bar{\ell}} \cup s^{\bar{\ell}}$ and satisfies $t_{\bar{\ell}}\left\lceil\zeta_{n} \in G_{n}^{\bar{\ell}}\right.$.

Proof. Since $P_{\zeta_{n+1}}$ is totally proper, the (countable) set $N_{\alpha_{0}} \cap \dot{G}_{\zeta_{n+1}}$ is forced to be an element of the ground model $V$. Thus the set of conditions that decide a particular value for $N_{\alpha_{0}} \cap \dot{G}_{\zeta_{n+1}}$ is a dense open subset of $P_{\zeta_{n+1}}$. In particular, the set

$$
D:=\left\{u \leq r_{\alpha_{0}}^{\bar{\ell}} \cup s^{\bar{\ell}}: u \text { decides a value for } N_{\alpha_{0}} \cap \dot{G}_{\zeta_{n+1}}\right\}
$$

is dense below $r_{\alpha_{0}}^{\bar{\ell}}$ in $P_{\zeta_{n+1}}$. Note that $D \in N_{\alpha_{0}+1}$ as all parameters needed to define $D$ are elements of $N_{\alpha_{0}+1}$.

It is easily seen that the set

$$
D \uparrow \zeta_{n}:=\left\{u \uparrow \zeta_{n}: u \in D\right\}
$$

is dense below $r_{\alpha_{0}}^{\bar{\ell}}$ in $P_{\zeta_{n}}$. Since $r_{\alpha_{0}}^{\bar{\ell}} \in G_{n}^{\bar{\ell}}$ and $D \uparrow \zeta_{n} \in N_{\alpha_{0}+1}$, it must be the case that the intersection $N_{\alpha_{0}+1} \cap \dot{G}_{n}^{\bar{\ell}} \cap D \uparrow \zeta_{n}$ is non-empty. Thus there is a condition $t_{\bar{\ell}} \in N_{\alpha_{0}+1} \cap D$ such that $t_{\bar{\ell}}\left\lceil\zeta_{n} \in G_{n}^{\bar{\ell}}\right.$.

Now why is $t_{\bar{\ell}}$ totally $\left(N_{\alpha_{0}}, P_{\zeta_{n+1}}\right)$-generic? We can find

$$
H \in \operatorname{Gen}\left(N_{\alpha_{0}}, P_{\zeta_{n+1}}\right) \cap N_{\alpha_{0}+1}
$$

such that

$$
t_{\bar{\ell}} \Vdash N_{\alpha_{0}} \cap \dot{G}_{\zeta_{n}}=H
$$

If $u \in H$, then $t_{\bar{\ell}}$ forces that $u$ is a member of $\dot{G}_{\zeta_{n}}$. Since without loss of generality all of our notions of forcing are separative, this means that $t_{\bar{\ell}}$ must be an extension of $u$. Thus $t_{\bar{\ell}}$ is a lower bound for $H$, and hence totally $\left(N_{\alpha_{0}}, P_{\zeta_{n+1}}\right)$-generic. 
Now we claim that $t_{\bar{\ell}}$ has all the required properties.

We have already ensured that $t_{\bar{\ell}} \leq r_{\alpha_{0}}^{\bar{\ell}} \cup t, t_{\bar{\ell}}$ is totally $\left(N_{\alpha_{0}}, P_{\zeta_{n+1}}\right)$-generic, and $t_{\bar{\ell}} \uparrow \zeta_{n} \in N_{\alpha_{0}+1} \cap G_{n}^{\bar{\ell}}$. Since $t_{\bar{\ell}} \leq r_{\alpha_{0}}^{\bar{\ell}} \cup s^{\bar{\ell}}$, we have that $t_{\bar{\ell}}$ is weakly $\left(\mathfrak{N} \uparrow J_{\bar{\ell}}, P_{\zeta_{n+1}}\right)-$ generic. Since $t_{\bar{\ell}}$ is totally $\left(N_{\alpha_{0}}, P_{\zeta_{n+1}}\right)$-generic, this implies

$$
J_{\bar{\ell}+1}:=\left\{\beta \in J_{\bar{\ell}}: t_{\bar{\ell}} \text { is totally }\left(N_{\beta}, P_{\zeta_{n+1}}\right) \text {-generic }\right\}
$$

has order-type $\alpha_{0}+1$. The fact that $J_{\bar{\ell}+1}$ is a suitable index set follows from the comments after the proof of Lemma 6.7

Once we have defined $t_{\bar{\ell}}$ and $J_{\bar{\ell}+1}$ for $\bar{\ell}<k_{n}$, we let $I_{n+1}=J_{k_{n}}$.

Goal: Find $s_{n+1}$.

Let us take a moment to assess our current situation. For each $\bar{\ell}<k_{n}$, we have a condition $t_{\bar{\ell}} \in N_{\alpha_{0}+1} \cap P_{\zeta_{n+1}}$ such that

- $t_{\bar{\ell}}$ is totally $\left(N_{\alpha_{0}}, P_{\zeta_{n+1}}\right)$-generic,

- $t_{\bar{\ell}}$ is weakly $\left(\mathfrak{N} \uparrow I_{n+1}, P_{\zeta_{n+1}}\right)$-generic,

- $t_{\bar{\ell}}\left\lceil\zeta_{n} \in G_{n}^{\bar{\ell}}\right.$.

We have also arranged that $I_{n+1}$ satisfies

- $\operatorname{otp}\left(I_{n+1}\right)=\alpha_{0}+1$,

- for each $\bar{\ell}<k_{n}, I_{n+1} \subseteq\left\{\beta \in I_{n}: t_{\bar{\ell}}\right.$ is totally $\left(N_{\beta}, P_{\zeta_{n+1}}\right)$-generic $\}$.

Since $\left\{t_{\bar{\ell}}: \bar{\ell}<k_{n}\right\} \subseteq N_{\alpha_{0}+1}$, we can patch these conditions together inside $N_{\alpha_{0}+1}$ to obtain a condition $t$ (essentially the disjunction of the conditions $t_{\ell}$ ) such that

- $t \in N_{\alpha_{0}+1} \cap P_{\zeta_{n+1}}$,

- for all $\ell<k, r^{\ell} \cup s_{n} \leq t \uparrow \zeta_{n}$,

- $t \Vdash\left(\exists \bar{\ell}<k_{n}\right)\left[t_{\bar{\ell}} \in \dot{G}_{\zeta_{n+1}}\right]$.

Given $t$, for each $\ell<k$ we apply Lemma 6.11 with $\zeta_{n}, \zeta_{n+1}, N_{\alpha_{0}+2}, N_{\alpha_{0}+3}, t$, and $r^{\ell} \cup s_{n}$ in place of the objects $i, j, N_{0}, N_{1}, p$, and $q$ appearing there.

Thus for each $\ell<k$, we get an object $u_{\ell}$ such that

- $r^{\ell} \cup s_{n} \cup u_{\ell} \in P_{\zeta_{n+1}}$,

- $r^{\ell} \cup s_{n} \cup u_{\ell} \leq t$,

- $r^{\ell} \cup s_{n} \cup u_{\ell}$ is $\left(N_{\alpha_{0}+2}, P_{\zeta_{n+1}}\right)^{\text {fin }}$-generic,

- $r^{\ell} \cup s_{n} \cup u_{\ell}$ is $\left(N_{\alpha_{0}+3}, P_{\zeta_{n+1}}\right)$-generic.

Now another patching argument amalgamates the (finitely many) $u_{\ell}$ 's to give us an $s_{n+1}$ such that for all $\ell<k$,

- $r^{\ell} \cup s_{n+1}$ and $r^{\ell} \cup s_{n} \cup u_{\ell}$ are equivalent as far as forcing with $P_{\zeta_{n+1}}$ is concerned,

- $r^{\ell} \cup s_{n+1}$ is $\left(N_{\alpha_{0}+2}, P_{\zeta_{n+1}}\right)^{\text {fin }}$-generic,

- $r^{\ell} \cup s_{n+1}$ is $\left(N_{\alpha_{0}+3}, P_{\zeta_{n+1}}\right)$-generic.

Note that we get $\left(N_{\alpha_{0}+2}, P_{\zeta_{n+1}}\right)$ fin - genericity because we have only finitely many $u_{\ell}$ 's.

Goal: Find $k_{n+1}$ and $\left\langle G_{n+1}^{\ell^{\prime}}: \ell^{\prime}<k_{n+1}\right\rangle$.

In the model $N_{\alpha_{0}+2}$, we have a $P_{\zeta_{n+1}}-$ name $\dot{\tau}$ for $N_{\alpha_{0}+1} \cap \dot{G}_{\zeta_{n+1}}$. Since $P_{\zeta_{n+1}}$ is totally proper,

$$
\Vdash_{P_{\zeta_{n+1}}} \dot{\tau} \in V .
$$

Since each $r^{\ell} \cup s_{n+1}$ is $\left(N_{\alpha_{0}+2}, P_{\zeta_{n+1}}\right)^{\text {fin }}$-generic, there is a finite set

$$
\mathcal{G} \subseteq \operatorname{Gen}\left(N_{\alpha_{0}+1}, P_{\zeta_{n+1}}\right) \cap N_{\alpha_{0}+2}
$$


such that

$$
(\forall \ell<k)\left[r^{\ell} \cup s_{n+1} \Vdash \dot{\tau} \in \mathcal{G}\right] .
$$

Without loss of generality, $|\mathcal{G}|$ is as small as possible and now we define $k_{n+1}=|\mathcal{G}|$ and list $\mathcal{G}$ as $\left\langle G_{n+1}^{\ell^{\prime}}: \ell^{\prime}<k_{n+1}\right\rangle$.

We still need to see that the sequence $\left\langle G_{n+1}^{\ell^{\prime}}: \ell^{\prime}\left\langle k_{n+1}\right\rangle\right.$ has the required attributes - in particular, we need properties (8), (9), and (10) of our induction hypothesis. Since $|\mathcal{G}|$ was taken to be as small as possible, our choice of $t$ implies that for all $\ell^{\prime}<k_{n+1}$, there exists $\bar{\ell}<k_{n}$ such that $t_{\bar{\ell}} \in G_{n+1}^{\ell^{\prime}}$, and this suffices to obtain the required properties.

Thus our induction can be carried out, and suitable objects found for each $n<$ $\omega$. To finish the proof of Proposition 6.13 for this case, note that the sequence $\left\{p_{n}: n \in \omega\right\}$ we constructed will generate a member $G^{*}$ of $\operatorname{Gen}\left(N_{0}, P_{\zeta}\right)$, which by choice of $p_{0}$ satisfies $p \in G^{*}$. If we define $s=\bigcup_{n<\omega} s_{n}$, then for each $\ell<k, r^{\ell} \cup s$ is a lower bound for $G^{*}$ in $P_{\zeta}$ — this is proved by showing that $r^{\ell} \cup s$ extends $p_{n}$ for each $n<\omega$. The requirement on the domain of $s$ causes no trouble either by the remark made after the statement of Lemma6.11

This completes the proof that Proposition 6.13 continues to hold for $\zeta$ a limit ordinal, and completes the proof by induction that Proposition 6.13 holds in all cases.

Now that Proposition 6.13 is done, it is quite easy to obtain the conclusion of Theorem 4 - we simply apply Proposition 6.13 to the case $\xi=0$ and $\zeta=\epsilon$. Proposition 6.13 is more than strong enough to allow us to prove that $P_{\epsilon}$ is totally proper.

\section{Constructing the MOdel}

In this section, we finally build a model of ZFC in which CH holds and every first countable, countably compact space is either compact, or contains a copy of $\omega_{1}$. Our construction has a fairly standard form - we do a countable support iteration of length $\omega_{2}$ over a model of GCH in which we kill every potential counterexample. We then appeal to the iteration theorem of the previous section to conclude that the Continuum Hypothesis remains true in the final generic extension.

The first thing we do is to sketch the proof of a lemma that has a right to be called folklore.

Lemma 7.1 (Folklore). Let $X$ be countably compact and countably tight. Then $X$ contains an uncountable free sequence if and only if $X$ contains a closed subset that is a closed pre-image of $\omega_{1}$.

Proof. Suppose first that $X$ contains an uncountable free sequence, i.e., a subset $F=\left\{x_{\alpha}: \alpha<\omega_{1}\right\}$ such that for each $\alpha<\omega_{1}$,

$$
\overline{\left\{x_{\beta}: \beta<\alpha\right\}} \cap \overline{\left\{x_{\beta}: \beta \geq \alpha\right\}}=\emptyset .
$$

For $\alpha<\omega_{1}$, we define $Y_{\alpha}=\overline{\left\{x_{\beta}: \beta<\alpha\right\}}$. Let $Y=\bigcup_{\alpha<\omega_{1}} Y_{\alpha}$, and we define a map $\pi: Y \rightarrow \omega_{1}$ by letting $\pi(x)$ be the least $\alpha$ such that $x \in Y_{\alpha}$.

Since $X$ is countably tight, it is clear that $Y$ is the closure of $F$ in $X$. It is also straightforward to verify that the map $\pi$ is continuous. The fact that $\pi$ is closed follows from the countable compactness of $X$. 
The converse has an even easier proof - let $x_{\alpha}$ be selected so that $\pi\left(x_{\alpha}\right)=\alpha+1$. Then the collection $\left\{x_{\alpha}: \alpha<\omega_{1}\right\}$ is a free sequence in $X$.

Proposition $7.2([6])$. Assume that $X$ is a first countable, countably compact, non-compact space that does not contain an uncountable free sequence. Then there is a totally proper, $<\omega_{1}-$ proper notion of forcing such that in the generic extension, $X$ acquires an uncountable free sequence.

Note that in the above proposition, since the notion of forcing is totally proper, $X$ will remain a first countable, countably compact non-compact space in the generic extension.

Proposition 7.3. Assume that $C H$ holds, and $X$ is a first countable, countably compact, non-compact Hausdorff space. Then there is a totally proper weakly $<\omega_{1}-$ proper notion of forcing $P_{X}$ such that in the $P_{X}$-generic extension, $X$ contains a closed subspace homeomorphic to $\omega_{1}$.

Proof. There are two cases.

CASE 1: $X$ contains a closed pre-image of $\omega_{1}$.

Since $X$ is first countable and the Continuum Hypothesis holds, it is straightforward to prove that $X$ contains a closed pre-image $Y$ of $\omega_{1}$ that is both closed in $X$ and of size $\aleph_{1}$. The notion of forcing detailed in section 3 of this paper (with $Y$ in place of the $X$ used there) then does the job - in the generic extension, $Y$ will still be a closed subset of $X$, and the forcing adjoins a closed subset $Z \subseteq Y$ homeomorphic to $\omega_{1}$.

CASE 2: $X$ does not contain a closed pre-image of $\omega_{1}$.

In this case, $P_{X}$ will be the composition $P * \dot{Q}$ of two totally proper notions of forcing. The first stage, $P$, will be the notion of forcing from [6] mentioned in Proposition 7.2. Since $P$ is totally proper, $X$ remains a first countable, countably compact non-compact space in the generic extension, and furthermore $X$ contains a closed pre-image of $\omega_{1}$. Since totally proper forcing adds no new reals, the Continuum Hypothesis remains true as well, so the next step is to proceed as in the first case to get a notion of forcing that adjoins a closed copy of $\omega_{1}$ to $X$. The composition of these two forcing notions is the $P_{X}$ that is required. (Note that the composition of a $<\omega_{1}$-proper notion of forcing and a weakly $<\omega_{1}$-proper notion of forcing is weakly $<\omega_{1}$-proper.)

Construction: We will define our iteration $\mathbb{P}=\left\langle P_{\xi}, \dot{Q}_{\xi}: \xi<\omega_{2}\right\rangle$ by induction on $\xi$. For this, it suffices to specify the identity of $\dot{Q}_{\xi}$ given $P_{\xi}$ as the fact that we are constructing a countable support iteration determines what happens at limit stages.

We assume that we have fixed ahead of time some reasonable bookkeeping procedure that will, at each stage $\xi$ of our construction, furnish us with (a $P_{\xi}$-name for) a first countable, countably compact, non-compact space $X$ of size $\aleph_{1}$ that must be taken care of. We also assume that the Continuum Hypothesis remains true after forcing with $P_{\xi}$. Given this, Proposition 7.3 tells us that there is a $P_{\xi}$-name $\dot{Q}_{\xi}$ for a totally proper notion of forcing that will give $X$ a closed subset homeomorphic to $\omega_{1}$.

Claim 7.4. Any countable support iteration $\mathbb{P}=\left\langle P_{\alpha}, \dot{Q}_{\alpha}: \alpha<\omega_{2}\right\rangle$ defined by the preceding recipe will satisfy the hypotheses of Theorem 4 . 
Proof. Our earlier work (and work done in [6]) tells us that for each $\alpha<\omega_{1}$,

$$
\Vdash_{P_{\alpha}} \dot{Q}_{\alpha} \text { is totally proper }
$$

and

$$
\Vdash_{P_{\alpha}} \dot{Q}_{\alpha} \text { is weakly }<\omega_{1} \text {-proper. }
$$

Thus we need only worry about the third hypothesis of Theorem 4 . Toward this goal, let us fix $\alpha$, and suppose we have objects $N_{0}, N_{1}, \bar{G}$, and $\left\langle G^{\ell}: \ell<k\right\rangle$ such that

- $N_{0}$ and $N_{1}$ are countable elementary submodels of $H(\lambda)$,

- $N_{0} \in N_{1}$,

- $\{\mathbb{P}, \alpha, \dot{q}\} \in N_{0}$,

- $\vdash_{P_{\alpha}} \dot{q} \in \dot{Q}_{\alpha}$

- $\bar{G} \in \operatorname{Gen}^{+}\left(N_{0}, P_{\alpha}, p\right) \cap N_{1}$,

- for $\ell<k, G^{\ell} \in \operatorname{Gen}\left(N_{1}, P_{\alpha}\right)$,

- for $\ell<k, \bar{G} \subseteq G^{\ell}$.

We will assume that our bookkeeping at stage $\alpha$ hands us a first countable closed pre-image of $\omega_{1}$ of size $\aleph_{1}$, so that $\dot{Q}_{\alpha}$ will be the notion of forcing detailed earlier in the paper. The other alternative for $\dot{Q}_{\alpha}$ can be handled by a similar argument in fact, in [6] the notion of forcing used is shown to satisfy an even more stringent requirement.

Let $\left\{\dot{D}_{n}: n \in \omega\right\} \in N_{1}$ be a list of all $P_{\alpha}$-names for dense subsets of $\dot{Q}_{\xi}$ that are elements of $N_{0}$. Our goal is to construct a sequence $\left\{\dot{p}_{n}: n \in \omega\right\}$ of $P_{\xi}$-names from $N_{0}$ such that

- $\bar{G} \Vdash \dot{p}_{n+1} \leq \dot{p}_{n}$,

- $\bar{G} \Vdash \dot{p}_{n+1} \in \dot{D}_{n}$,

- for each $\ell<k, G^{\ell} \Vdash\left\{\dot{p}_{n}: n \in \omega\right\}$ has a lower bound.

Before we do this, we reflect for a moment on the proof of Theorem 1. We constructed a decreasing sequence of conditions $\left\{p_{n}: n \in \omega\right\}$ that hit every dense subset of $P$ that is a member of $N$, and we had to take action to ensure that the sequence had a lower bound. The action we took was, essentially, to make sure that the sequence of sets $\left[p_{n+1}\right] \backslash\left[p_{n}\right]$ converged "fast enough" to a point in $\operatorname{Tr}(N)-$ rapid enough convergence makes sure that the sequence has a lower bound. In our current situation, we will have to make sure that each $G^{\ell}$ thinks that the sequence $\left[\dot{p}_{n+1}\right] \backslash\left[\dot{p}_{n}\right]$ converges "fast enough" so that we will end up with a lower bound no matter which $G^{\ell}$ turns out to be $N_{1} \cap \dot{G}$.

So how do we do this? In $N_{0}$, there are $P_{\alpha}$-names $\dot{X}$ and $\dot{\mathcal{U}}$ for the topological space and filter of closed subsets under consideration. Let $\left\{\dot{D}_{n}: n \in \omega\right\} \in N_{1}$ be as above.

Claim 7.5. In $N_{1}$ there are objects $\dot{z}$ and $\left\langle\dot{x}_{n}: n \in \omega\right\rangle$ such that

(1) $\dot{z} \in N_{1}^{P_{\alpha}}$ is a name for a point in $\dot{X}$,

(2) each $\dot{x}_{n}$ is a name in $N_{0}$ for a point in $\dot{X}$,

(3) if $\dot{A} \in N_{0}^{P_{\alpha}}$ is a name for an element of $\dot{\mathcal{U}}$, then for all sufficiently large $n$, $\bar{G} \Vdash \dot{x}_{n} \in \dot{A}$,

(4) for all $\ell<k, G^{\ell} \Vdash\left\langle\dot{x}_{n}: n \in \omega\right\rangle$ converges to $\dot{z}$. 
Proof. We work inside the model $N_{1}$. Let $\left\{\dot{A}_{n}: n \in \omega\right\}$ list all names in $N_{0}^{P_{\alpha}}$ for elements of $\dot{\mathcal{U}}$. We know that for each $n<\omega$,

$$
\bar{G} \Vdash \bigcap_{i<n} \dot{A}_{i} \neq \emptyset,
$$

and so for each $n$ we can find a name $\dot{x}_{n} \in N_{0}^{P_{\alpha}}$ such that

$$
\bar{G} \Vdash \dot{x}_{n} \in \dot{A}_{0} \cap \cdots \cap \dot{A}_{n-1} .
$$

This produces a sequence $\left\langle\dot{x}_{n}: n \in \omega\right\rangle$ satisfying requirement (3) of the claim, and since we were working in $N_{1}$, without loss of generality $\left\langle\dot{x}_{n}: n \in \omega\right\rangle \in N_{1}$.

$\dot{X}$ is (forced to be) a closed pre-image of $\omega_{1}$, and so by our choice of $\left\langle\dot{x}_{n}: n \in \omega\right\rangle$ we know there are names $\dot{I}_{0}$ and $\dot{z}_{0}$ in $N_{1}$ such that

$$
G^{0} \Vdash\left\langle\dot{x}_{n}: n \in \dot{I}_{0}\right\rangle \text { converges to } \dot{z}_{0} \text {. }
$$

Now $\dot{I}_{0}$ is a name for a subset of $\omega$ and $P_{\alpha}$ is totally proper, so there is an honestto-goodness $I_{0} \subseteq \omega$ such that

$$
G^{0} \Vdash \dot{I}_{0}=I_{0} .
$$

Note that $I_{0} \in N_{1}$, hence the sequence $\left\langle\dot{x}_{n}: n \in I_{0}\right\rangle$ is in $N_{1}$ as well. By re-indexing, we achieve that there is a name $\dot{z}_{0} \in N_{1}$ such that

$$
G^{0} \Vdash\left\langle\dot{x}_{n}: n \in \omega\right\rangle \text { converges to } \dot{z}_{0} .
$$

Now we repeat the same argument, running through the rest of the $G^{\ell}$ 's. Since there are only finitely many $G^{\ell}$ 's, we are left with a sequence $\left\langle\dot{x}_{n}: n \in \omega\right\rangle$ and names $\left\langle\dot{z}_{\ell}: \ell<k\right\rangle$ such that

$$
G^{\ell} \Vdash\left\langle\dot{x}_{n}: n \in \omega\right\rangle \text { converges to } \dot{z}_{\ell} .
$$

A standard argument allows us to replace the names $\dot{z}_{0}, \ldots, \dot{z}_{k-1}$ with a single name $\dot{z}$ - again, since we have only finitely many names to patch together, we can assume $\dot{z} \in N_{1}$. We finish by noting that requirement (3) is preserved by passing to infinite subsequences.

Note that for each $\ell<k$, we have

$$
G^{\ell} \Vdash \dot{z} \in \operatorname{Tr}\left(N_{0}\left[\dot{G}_{\alpha}\right]\right) ;
$$

this follows by our choice of $\left\langle\dot{x}_{n}: n \in \omega\right\rangle$. Let $\left\{\dot{U}_{n}: n \in \omega\right\}$ be a sequence of $P_{\alpha^{-}}$ names such that every condition forces that the interpretations of the $\dot{U}_{n}$ 's form a decreasing base of open neighborhoods for $\dot{z}$.

We will define by induction on $n$ a sequence $\left\{\dot{p}_{n}: n \in \omega\right\}$ and function $h \in{ }^{\omega} \omega$ such that

(1) $\dot{p}_{0}=\dot{q}, h(0)=0$,

(2) $\dot{p}_{n} \in N_{0}^{P}\left(\subseteq N_{1}\right)$,

(3) $\bar{G} \Vdash \dot{p}_{n+1} \leq \dot{p}_{n}$,

(4) $\bar{G} \Vdash \dot{p}_{n+1} \in \dot{D}_{n}$,

(5) $h(n)<h(n+1)$,

(6) for each $\ell<k, G^{\ell} \Vdash\left[\dot{p}_{n+1}\right] \backslash\left[\dot{p}_{n}\right] \subseteq \dot{U}_{h(n+1)}$,

(7) if $\dot{f}$ is a name in $N_{0}^{P}$ such that $\bar{G} \Vdash \dot{f} \in \Phi_{\dot{p}_{i}}$ for some $i$, then there is a stage $n \geq i$ such that for each $\ell<k$,

$$
G^{\ell} \Vdash\left\{x \in Y\left(\dot{f}, \dot{p}_{n}, \dot{p}_{i}\right): \dot{U}_{h(n+1)} \subseteq \dot{f}(x)\right\} \text { is large. }
$$


Just as in the proof of Theorem 1 we assume that some bookkeeping procedure has been fixed at the start so that at each stage we will be handed a (name for a) promise $\dot{f}$ that must be taken care of. Assume we are given $p_{n}$ and $h\lceil n+1$ and that our bookkeeping hands us a $\dot{f}$ such that for some $i \leq n$,

$$
\bar{G} \Vdash \dot{f} \in \Phi_{\dot{p}_{i}} .
$$

In $N_{0}^{P}$, we can find a $P_{\alpha}$-name $\dot{f}^{\prime}$ such that

$$
\bar{G} \Vdash \dot{f}^{\prime}=\dot{f} \uparrow Y\left(\dot{f}, \dot{p}_{n}, \dot{p}_{i}\right) .
$$

Fix $\ell<k$. We know

$$
G^{\ell} \Vdash \dot{z} \notin \operatorname{Ban} \dot{f}^{\prime},
$$

and so there is a natural number $m_{\ell}$ such that

$$
G^{\ell} \Vdash\left\{x \in Y\left(\dot{f}, \dot{p}_{n}, \dot{p}_{i}\right): \dot{U}_{m_{\ell}} \subseteq \dot{f}(x)\right\} \text { is large. }
$$

We define $h(n+1)$ to be the least number greater than $h(n)$ and all $m_{\ell}$ 's for $\ell<k$. With this choice of $h(n+1)$, we have that for each $\ell<k$,

$$
G^{\ell} \Vdash\left\{x \in Y\left(\dot{f}, \dot{p}_{n}, \dot{p}_{i}\right): \dot{U}_{h(n+1)} \subseteq \dot{f}(x)\right\} \text { is large. }
$$

Note that we have taken care of this particular instance of requirement (7.7). Next, we choose $i_{n}<\omega$ large enough so that for all $\ell<k$,

$$
G^{\ell} \Vdash \dot{x}_{i_{n}} \in \dot{U}_{h(n+1)} .
$$

This is possible because there are only finitely many $G^{\ell}$ 's to worry about.

The model $N_{0}$ will contain names for all the members of a countable neighborhood base of $\dot{x}_{i_{n}}$, so we can find $\dot{V} \in N_{0}^{P_{\alpha}}$ such that

$$
\bar{G} \Vdash \dot{V} \text { is a neighborhood of } \dot{x}_{i_{n}}
$$

and

$$
(\forall \ell<k) G^{\ell} \Vdash \dot{V} \subseteq \dot{U}_{h(n+1)} .
$$

Now choose $\dot{p}_{n+1} \in N_{0}^{P_{\alpha}}$ such that $\bar{G}$ forces $\dot{p}_{n+1} \leq \dot{p}_{n}, \dot{p}_{n+1} \in \dot{D}_{n}$, and $\left[\dot{p}_{n+1}\right] \backslash$ $\left[\dot{p}_{n}\right] \subseteq \dot{V}$. All this is possible by our work earlier in the paper, and just as in the proof of Theorem 1, we have

$$
(\forall \ell<k) G^{\ell} \Vdash\left\{\dot{p}_{n}: n \in \omega\right\} \text { has a lower bound, }
$$

as required.

Now that we have established that our construction satisfies the assumptions of our iteration theorem, the rest of the proof follows standard lines.

Theorem 5. It is consistent that the Continuum Hypothesis holds and that first countable, countably compact regular spaces are either compact or contain a closed copy of $\omega_{1}$.

Proof. We start with a model of $\mathrm{ZFC}+\mathrm{CH}$, and force with an iteration $\mathbb{P}=\left\langle P_{\alpha}, \dot{Q}_{\alpha}\right.$ : $\left.\alpha<\omega_{2}\right\rangle$ as described earlier in this section. We have shown that any such iteration satisfies the hypotheses of our iteration theorem, and so the limit forcing $P_{\omega_{2}}$ is totally proper. It is also easy to see that all iterands of $\mathbb{P}$ are $\omega_{2}$-centered, and so $P_{\omega_{2}}$ will preserve all cardinals and cofinalities. A standard argument lets us construct $\mathbb{P}$ so that every first countable, countably compact, non-compact space of size $\aleph_{1}$ is "taken care of" at some stage of the iteration, i.e., every such space contains a 
closed copy of $\omega_{1}$ in $V\left[G_{\omega_{2}}\right]$. Assuming CH holds, every first countable, countably compact, non-compact space contains a closed non-compact subset of size $\aleph_{1}$. Since CH holds in $V\left[G_{\omega_{2}}\right]$, we see that in our model, every first countable, countably compact, non-compact $T_{3}$ space contains a (closed) subset homeomorphic to $\omega_{1}$.

\section{What's SPECIAL ABOUT $\omega_{1}$ ?}

In this section, we prove in ZFC that there is a two-to-one closed pre-image of $\omega_{2}$ that does not contain a copy of $\omega_{2}$. We use Shelah's method of club-guessing - the references for this are [18] and [17] of [16].

We start by fixing some notation.

Definition 8.1. If $\lambda$ and $\mu$ are regular cardinals, then we define

$$
S_{\mu}^{\lambda}=\{\delta<\lambda: \operatorname{cf}(\delta)=\mu\} .
$$

By Claim III.2.3 of [16], there is a family

$$
\bar{C}=\left\langle C_{\delta}: \delta \in S_{\aleph_{0}}^{\aleph_{2}}\right\rangle
$$

such that each $C_{\delta}$ is cofinal in $\delta, C_{\delta}$ has order-type $\omega$, and for every closed unbounded $C \subseteq \omega_{2}$, there is a stationary set of $\delta$ 's such that $C_{\delta} \backslash C$ is finite.

For $\delta \in S_{\aleph_{0}}^{\aleph_{2}}$, let $\eta_{\delta}: \omega \rightarrow \delta$ be the increasing enumeration of $C_{\delta}$.

We will obtain the underlying set of our space $X$ by taking $\omega_{2}$ and "doubling" the points of cofinality $\omega$, i.e., the underlying set of $X$ is $\left(\omega_{2} \backslash S_{\aleph_{0}}^{\aleph_{2}}\right) \cup\left(S_{\aleph_{0}}^{\aleph_{2}} \times\{0,1\}\right)$. We will topologize $X$ in such a way that the natural projection $\pi: X \rightarrow \omega_{2}$ becomes a closed map.

For $\alpha<\omega_{2}$, by "level $\alpha$ " we mean the set $\pi^{-1}(\{\alpha\})$. Each level of our space has cardinality at most 2 . We will define the topology by induction on the level of the points in $X$.

To start, the first level of our space (i.e., level 0) is an isolated point.

Our induction hypothesis for stage $\alpha$ is that we have topologized $\pi^{-1}(\alpha)$ in such a way that the restriction of $\pi$ to this initial segment of $X$ is a closed mapping.

If $\alpha$ is a successor ordinal, the level $\alpha$ consists of a single point, and we declare that this point is isolated in $X$.

If $\alpha$ is a limit ordinal of cofinality $\omega_{1}$, then level $\alpha$ consists of a single point, and basic open neighborhoods of this point will consist of sets of the form $\pi^{-1}([\beta+1, \alpha])$, where $\beta<\alpha$.

The work comes in when $\alpha$ is a limit ordinal of countable cofinality. Let $x_{0}$ and $x_{1}$ denote the two points in $X$ at level $\alpha$.

We will arrange that $x_{0}$ and $x_{1}$ each have a countable base in $X$. A basic open neighborhood of $x_{0}$ is of the form

$$
\bigcup_{n \geq m} \pi^{-1}\left(\left[\eta_{\alpha}(2 n)+1, \eta_{\alpha}(2 n+1)\right]\right)
$$

for $m<\omega$, while a basic open neighborhood of $x_{1}$ is of the form

$$
\bigcup_{n \geq m} \pi^{-1}\left(\left[\eta_{\alpha}(2 n+1)+1, \eta_{\alpha}(2 n+2)\right]\right) \text {. }
$$

In plain language, we use $C_{\alpha}$ to partition $\alpha$ into an $\omega$-sequence of intervals. A basic open neighborhood of $x_{0}$ looks like the pre-image (under $\pi$ ) of a tail of the "even intervals", while a basic open neighborhood of $x_{1}$ is similar with even replaced by odd. 
It is not hard to verify that this defines a topology on $X$ with the advertised bases at each point, and also that the mapping $\pi$ is continuous and closed.

Proposition 8.2. $X$ does not contain a closed set homeomorphic to $\omega_{2}$.

Proof. We work by contradiction. Assume $Y \subseteq X$ is homeomorphic to $\omega_{2}$, witnessed by the homeomorphism $f$. Since $\pi$ is a closed mapping, we know the set $C:=\pi[Y]$ is closed and unbounded in $\omega_{2}$.

Let $M$ be an elementary submodel of $H(\lambda)$ for some large $\lambda$ satisfying

- $\{X, Y, \bar{C}, f\} \in M$,

- $M \cap \omega_{2}$ is an ordinal of cofinality $\omega$,

- if $\delta=M \cap \omega_{2}$, then $C_{\delta} \backslash C$ is finite.

Such an $M$ can be found as the set of such $\delta$ 's is stationary in $\omega_{2}$.

Note that $M \cap Y=f^{-1}(\delta)$, and this means that the $\overline{M \cap Y} \backslash(M \cap Y)$ consists of the singleton $f^{-1}(\{\delta\})$. We will get a contradiction by showing that $\pi^{-1}(\{\delta\}) \subseteq$ $\overline{M \cap Y}$.

We show that $x_{0} \in \overline{M \cap Y}$; the proof that $x_{1} \in \overline{M \cap Y}$ is entirely analogous.

Let $U$ be a basic open neighborhood of $x_{0}$. We need only show that $U \cap Y \neq \emptyset$.

By definition, there is an $m$ such that

$$
U=\bigcup_{n \geq m} \pi^{-1}\left(\left[\eta_{\delta}(2 n)+1, \eta_{\delta}(2 n+1)\right]\right) .
$$

In particular,

$$
\bigcup_{n \geq m} \pi^{-1}\left(\left\{\eta_{\delta}(2 n+1)\right\}\right) \subseteq U .
$$

For all large enough $k$, we know

$$
Y \cap \pi^{-1}\left(\left\{\eta_{\delta}(k)\right\}\right) \neq \emptyset,
$$

as $\eta_{\delta}(k) \in C=\pi[Y]$. This means that $U \cap Y \neq \emptyset$ as desired. We therefore have a contradiction, and the proposition is established.

\section{REFERENCES}

[1] Z. Balogh, A. Dow, D. H. Fremlin, and P. J. Nyikos, Countable tightness and proper forcing, Bull. Amer. Math. Soc. (N.S.) 19 (1988), no. 1, 295-298. MR.89e:03088

[2] Zoltán Balogh, On compact Hausdorff spaces of countable tightness, Proc. Amer. Math. Soc. 105 (1989), no. 3, 755-764. MR89h:03088

[3] Alan Dow, An introduction to applications of elementary submodels to topology, Topology Proc. 13 (1988), no. 1, 17-72. MR91a:54003

[4] - Set theory in topology, Recent progress in general topology (Prague, 1991), NorthHolland, Amsterdam, 1992, pp. 167-197. MR1229121 (95g:54004)

[5] - More set-theory for topologists, Topology Appl. 64 (1995), no. 3, 243-300. MR 97a:54005

[6] Todd Eisworth, $\mathrm{CH}$ and first countable, countably compact spaces, Topology Appl. 109 (2001), no. 1, 55-73. MR2001k:54010

[7] Todd Eisworth, On perfect pre-images of $\omega_{1}$, Topology Appl. 125 (2002), no. 2, 263-278. MR.1933576 (2003g:54005)

[8] - Totally proper forcing and the Moore-Mrowka problem, Fund. Math. 177 (2003), no. 2, 121-137. MR.1992528 (2004g:03093)

[9] Todd Eisworth and Judith Roitman, CH with no Ostaszewski spaces, Trans. Amer. Math. Soc. 351 (1999), no. 7, 2675-2693. MR2000b:03182

[10] Ryszard Engelking, General topology, Heldermann Verlag, Berlin, 1989. MR 91c:54001 
[11] D. H. Fremlin, Perfect pre-images of $\omega_{i}$ and the PFA, Topology Appl. 29 (1988), no. 2, 151-166. MR89i:03096

[12] Martin Goldstern, Tools for your forcing construction, Set theory of the reals (Ramat Gan, 1991), Bar-Ilan Univ., Ramat Gan, 1993, pp. 305-360. MF 94h:03102

[13] Gary Gruenhage, Spaces having a small diagonal, Proceedings of the International Conference on Topology and its Applications (Yokohama, 1999). Topology Appl. 122 (2002), no. 1-2, 183-200. MR 1919300 (2003g:54050)

[14] Peter Nyikos, The theory of nonmetrizable manifolds, Handbook of set-theoretic topology, North-Holland, Amsterdam, 1984, pp. 633-684. MR86f:54054

[15] Saharon Shelah, Nnr revisited, Journal of Symbolic Logic.

[16] _ Cardinal arithmetic, Oxford Logic Guides, vol. 29, Oxford University Press, 1994. MR 1318912 (96e:03001)

[17] Jonsson Algebras in an inaccessible $\lambda$ not $\lambda$-Mahlo, Cardinal Arithmetic, Oxford Logic Guides, vol. 29, Oxford University Press, 1994.

[18] - There are Jonsson algebras in many inaccessible cardinals, Cardinal Arithmetic, Oxford Logic Guides, vol. 29, Oxford University Press, 1994.

[19] - Proper and improper forcing, Perspectives in Mathematical Logic, Springer, 1998. MR.1623206 (98m:03002)

[20] Jerry E. Vaughan, Countably compact and sequentially compact spaces, Handbook of settheoretic topology, North-Holland, Amsterdam, 1984, pp. 569-602. MR 86c:54022

Department of Mathematics, University of Northern Iowa, Cedar Falls, Iowa 50613 E-mail address: eisworth@uni.edu

Current address: Department of Mathematics, Ohio University, Athens, Ohio 45701

E-mail address: eisworth@math.ohiou.edu

Department of Mathematics, University of South Carolina, Columbia, South CarOLINA 29208

E-mail address: nyikos@math.sc.edu 